\title{
Two-Level Iteration Penalty Methods for the Navier-Stokes Equations with Friction Boundary Conditions
}

\author{
Yuan Li and Rong An \\ College of Mathematics and Information Science, Wenzhou University, Wenzhou 325035, China \\ Correspondence should be addressed to Rong An; anrong702@gmail.com
}

Received 2 April 2013; Revised 12 June 2013; Accepted 19 June 2013

Academic Editor: Stanislaw Migorski

Copyright ( 2013 Y. Li and R. An. This is an open access article distributed under the Creative Commons Attribution License, which permits unrestricted use, distribution, and reproduction in any medium, provided the original work is properly cited.

\begin{abstract}
This paper presents two-level iteration penalty finite element methods to approximate the solution of the Navier-Stokes equations with friction boundary conditions. The basic idea is to solve the Navier-Stokes type variational inequality problem on a coarse mesh with mesh size $H$ in combining with solving a Stokes, Oseen, or linearized Navier-Stokes type variational inequality problem for Stokes, Oseen, or Newton iteration on a fine mesh with mesh size $h$. The error estimate obtained in this paper shows that if $H, h$, and $\varepsilon$ can be chosen appropriately, then these two-level iteration penalty methods are of the same convergence orders as the usual one-level iteration penalty method.
\end{abstract}

\section{Introduction}

In this paper, we consider a two-level iteration penalty method for the incompressible flows which are governed by the incompressible Navier-Stokes equations:

$$
\begin{gathered}
-\mu \Delta \mathbf{u}+(\mathbf{u} \cdot \nabla) \mathbf{u}-\nabla p=\mathbf{f}, \quad \text { in } \Omega, \\
\operatorname{div} \mathbf{u}=0, \quad \text { in } \Omega,
\end{gathered}
$$

where $\Omega$ is a bounded domain in $\mathbb{R}^{2}$ assumed to have a Lipschitz continuous boundary $\partial \Omega, \mu>0$ represents the viscous coefficient, $\mathbf{u}=\left(u_{1}(x), u_{2}(x)\right)$ denotes the velocity vector, $p=p(x)$ the pressure and $\mathbf{f}=\left(f_{1}(x), f_{2}(x)\right)$ the prescribed body force vector. The solenoidal condition $\operatorname{div} \mathbf{u}=0$ means that the flows are incompressible.

Instead of the classical whole homogeneous boundary conditions, here we consider the following slip boundary conditions with friction type:

$$
\begin{gathered}
\mathbf{u}=0, \quad \text { on } \Gamma, \\
\mathbf{u}_{n}=0, \quad-\sigma_{\tau}(\mathbf{u}) \in g \partial\left|\mathbf{u}_{\tau}\right| \text { on } S,
\end{gathered}
$$

where $\Gamma \cap S=\emptyset, \overline{\Gamma \cup S}=\partial \Omega$, and $g$ is a scalar function; $\mathbf{u}_{n}=\mathbf{u} \cdot \mathbf{n}$ and $\mathbf{u}_{\tau}=\mathbf{u}-\mathbf{u}_{n} \mathbf{n}$ are the normal and tangential components of the velocity, where $\mathbf{n}$ stands for the unit vector of the external normal to $S ; \sigma_{\tau}(\mathbf{u})=\sigma-\sigma_{n} \mathbf{n}$, independent of $p$, is the tangential components of the stress vector $\sigma$ which is defined by $\sigma_{i}=\sigma_{i}(\mathbf{u}, p)=\left(\mu e_{i j}(\mathbf{u})-p \delta_{i j}\right) n_{j}$ with $e_{i j}(\mathbf{u})=\left(\partial u_{i} / \partial x^{j}\right)+\left(\partial u_{j} / \partial x^{i}\right), i, j=1,2$. The set $\partial \psi(\mathbf{a})$ denotes a subdifferential of the function $\psi$ at $\mathbf{a} \in L^{2}(S)^{2}$, whose definition will be given in the next section.

This type of boundary condition is firstly introduced by Fujita [1] where some problems in hydrodynamics are studied. Some theoretical problems are also studied by many scholars, such as Fujita in [2-4], Y. Li and K. Li $[5,6]$, and Saito and Fujita $[7,8]$ and references cited in their work.

The development of appropriate mixed finite element approximations is a key component in the search for efficient techniques for solving the problem (1) quickly and efficiently. Roughly speaking, there exist two main difficulties. One is the nonlinear term $(\mathbf{u} \cdot \nabla) \mathbf{u}$, which can be processed by the linearization method such as the Newton iteration method, Stokes iteration method, Oseen iteration method [9], or the two-level methods [10-17]. The other is that the velocity and the pressure are coupled by the solenoidal condition. The popular technique to overcome the second difficulty is to relax the solenoidal condition in an appropriate method and to result in a pseudocompressible system, such as the penalty method and the artificial compressible method [18]. Recently, using the Taylor-Hood element, the authors [19] study the 
penalty finite element method for the problem (1)-(2). Denote $\left(\mathbf{u}_{\varepsilon}^{h}, p_{\varepsilon}^{h}\right)$ as the penalty finite element approximation solution to $(\mathbf{u}, p) \in\left(H^{3}(\Omega)^{2}, H^{2}(\Omega)\right)$. The error estimate derived in [19] is

$$
\left\|\mathbf{u}-\mathbf{u}_{\varepsilon}^{h}\right\|_{1}+\left\|p-p_{\varepsilon}^{h}\right\| \leq c\left(\varepsilon+h^{5 / 4}\right),
$$

where $\varepsilon>0$ is the penalty parameter. However, the condition number of the numerical discretization for the penalty methods is $O\left(\varepsilon^{-1} h^{-2}\right)$, which will result in an illconditioned problem when mesh size $h \rightarrow 0$. In order to avoid the choice of the small parameter $\varepsilon$, Dai et al. [20] have studied the iteration penalty finite element method and derive

$$
\left\|\mathbf{u}-\mathbf{u}_{\varepsilon}^{h k}\right\|_{1}+\left\|p-p_{\varepsilon}^{h k}\right\| \leq c\left(\varepsilon^{k+1}+h^{5 / 4}\right)
$$

where $k \in \mathbb{N}^{+}$is the iteration step number.

In this paper, we combine the iteration penalty method with the two-level method to approximate the solution of the problem (1)-(2). The iterative penalty method was first introduced by Cheng and Shaikh [21] for the Stokes equations and further used to solve the pure Neumann problem [22]. This iteration penalty method can be considered as the time discretization of the artificial compressible method [23]. The two-level iteration penalty methods studied in this paper can be described as follows. The first step and the second step are required to solve a small Navier-Stokes equations on the coarse mesh in terms of the iteration penalty method $[20,21]$. The third step is required to solve a large linearization problem on the fine mesh in terms of the Stokes iteration, Oseen iteration, or Newtonian iteration, respectively. We prove that these two-level iteration penalty finite element solutions $\left(\mathbf{u}_{\varepsilon h}, p_{\varepsilon h}\right)$ are of the following error estimate:

$$
\begin{aligned}
\| \mathbf{u}- & \mathbf{u}_{\varepsilon h}\left\|_{1}+\right\| p-p_{\varepsilon h} \| \\
\leq & \left\{\begin{array}{r}
\left(h^{5 / 4}+H^{9 / 4}+\varepsilon H^{5 / 4}+\varepsilon^{k+1}\right), \\
c\left(h^{5 / 4}+H^{5 / 2}+\varepsilon H^{5 / 4}+\varepsilon^{k+2}\right), \\
\text { (Newtonian iteration) },
\end{array}\right.
\end{aligned}
$$

Finally, we propose an improved correction iteration scheme for $\left(u_{\varepsilon h}, p_{\varepsilon h}\right)$ in terms of the Newton iteration method. We prove that the correction finite element solutions $\left(u_{\varepsilon h}^{\star}, p_{\varepsilon h}^{\star}\right)$ are of the following error estimates:

$$
\begin{aligned}
& \left\|\mathbf{u}-\mathbf{u}_{\varepsilon h}^{\star}\right\|_{1}+\left\|p-p_{\varepsilon h}^{\star}\right\| \\
& \leq\left\{\begin{array}{r}
c\left(h^{5 / 4}+H^{9 / 2}+\varepsilon^{3 / 2} H^{5 / 4}+\varepsilon^{1 / 2} H^{9 / 4}+\varepsilon^{k+1 / 2}\right), \\
(\text { Stokes and Oseen iteration) }, \\
c\left(h^{5 / 4}+H^{5}+\varepsilon^{3 / 2} H^{5 / 4}+\varepsilon^{1 / 2} H^{5 / 2}+\varepsilon^{k+1 / 2}\right), \\
\text { (Newtonian iteration) } .
\end{array}\right.
\end{aligned}
$$

Throughout this paper, we will use $c$ to denote a positive constant whose value may change from place to place but that remains independent of $h, H$, and $\varepsilon$ and that may depend on $\mu, \Omega$ and the norms of $\mathbf{u}, p, \mathbf{f}$, and $g$.

\section{Preliminary}

First, we give the definition of the subdifferential property. Let $\psi$ be a given function possessing the properties of convexity and weak semicontinuity from below. We say that the set $\partial \psi(\mathbf{a})$ is a subdifferential of the function $\psi$ at $\mathbf{a} \in L^{2}(S)^{2}$ if and only if

$$
\begin{aligned}
\partial \psi(\mathbf{a})=\{\mathbf{b} & \in L^{2}(S)^{2}: \psi(\mathbf{h})-\psi(\mathbf{a}) \\
& \left.\geq \mathbf{b} \cdot(\mathbf{h}-\mathbf{a}), \quad \forall \mathbf{h} \in L^{2}(S)^{2}\right\} .
\end{aligned}
$$

In what follows, we employ the standard notation $H^{l}(\Omega)$ (or $H^{l}(\Omega)^{2}$ ) and $\|\cdot\|_{l}, l \geq 0$, for the Sobolev spaces of all functions having square integrable derivatives up to order $l$ in $\Omega$ and the standard Sobolev norm. When $l=0$, we write $L^{2}(\Omega)\left(\right.$ or $\left.L^{2}(\Omega)^{2}\right)$ and $\|\cdot\|$ instead of $H^{0}(\Omega)\left(\right.$ or $\left.H^{0}(\Omega)^{2}\right)$ and $\|\cdot\|_{0}$, respectively.

For the mathematical setting, we introduce the following spaces:

$$
\begin{gathered}
V=\left\{\mathbf{u} \in H^{1}(\Omega)^{2},\left.\mathbf{u}\right|_{\Gamma}=0,\left.\mathbf{u} \cdot \mathbf{n}\right|_{S}=0\right\}, \\
V_{0}=H_{0}^{1}(\Omega)^{2}, \\
V_{\sigma}=\{\mathbf{u} \in V, \operatorname{div} \mathbf{u}=0\}, \\
M=L_{0}^{2}(\Omega)=\left\{q \in L^{2}(\Omega), \int_{\Omega} q d x=0\right\} .
\end{gathered}
$$

The space $V$ is equipped with the norm

$$
\|\mathbf{v}\|_{V}=\left(\int_{\Omega}|\nabla \mathbf{v}|^{2} d x\right)^{1 / 2} .
$$

It is well known that $\|\mathbf{v}\|_{V}$ is equivalent to $\|\mathbf{v}\|_{1}$ due to Poincare's inequality. Introduce two bilinear forms

$$
\begin{gathered}
a(\mathbf{u}, \mathbf{v})=\mu \int_{\Omega} \nabla \mathbf{u} \cdot \nabla \mathbf{v} d x, \quad \forall \mathbf{u}, \mathbf{v} \in V, \\
d(v, q)=\int_{\Omega} q \operatorname{div} \mathbf{v} d x, \quad \forall \mathbf{v} \in V, q \in M,
\end{gathered}
$$

and a trilinear form

$$
\begin{aligned}
b(\mathbf{u}, \mathbf{v}, \mathbf{w}) & =\int_{\Omega}(\mathbf{u} \cdot \nabla) \mathbf{v} \cdot \mathbf{w} d x-\frac{1}{2} \int_{\Omega} \operatorname{div} \mathbf{u v} \cdot \mathbf{w} d x \\
& =\frac{1}{2} \int_{\Omega}(\mathbf{u} \cdot \nabla) \mathbf{v} \cdot \mathbf{w} d x-\frac{1}{2} \int_{\Omega}(\mathbf{u} \cdot \nabla) \mathbf{w} \cdot \mathbf{v} d x .
\end{aligned}
$$

It is easy to verify that this trilinear form satisfies the following important properties $[12,23]$ :

$$
\begin{gathered}
b(\mathbf{u}, \mathbf{v}, \mathbf{w})=-b(\mathbf{u}, \mathbf{w}, \mathbf{v}), \\
b(\mathbf{u}, \mathbf{v}, \mathbf{w}) \leq N\|\mathbf{u}\|_{V}\|\mathbf{v}\|_{V}\|\mathbf{w}\|_{V},
\end{gathered}
$$

$$
\begin{aligned}
b(\mathbf{u}, \mathbf{v}, \mathbf{w}) \leq & \frac{N}{2}\|\mathbf{u}\|^{1 / 2}\|\mathbf{u}\|_{V}^{1 / 2} \\
& \times\left(\|\mathbf{v}\|_{V}\|\mathbf{w}\|^{1 / 2}\|\mathbf{w}\|_{V}^{1 / 2}+\|\mathbf{w}\|_{V}\|\mathbf{v}\|^{1 / 2}\|\mathbf{v}\|_{V}^{1 / 2}\right),
\end{aligned}
$$


for all $\mathbf{u}, \mathbf{v}, \mathbf{w} \in V$, and

$|b(\mathbf{u}, \mathbf{v}, \mathbf{w})|+|b(\mathbf{v}, \mathbf{u}, \mathbf{w})|+|b(\mathbf{w}, \mathbf{u}, \mathbf{v})| \leq N\|\mathbf{u}\|_{V}\|\mathbf{v}\|_{2}\|\mathbf{w}\|$,

for all $\mathbf{u} \in V, \mathbf{v} \in H^{2}(\Omega)^{2}$, and $\mathbf{w} \in L^{2}(\Omega)^{2}$, where $N>0$ depends only on $\Omega$.

Given $\mathbf{f} \in L^{2}(\Omega)^{2}$ and $g \in L^{2}(S)$ with $g>0$ on $S$, under the above notation, the variational formulation of the problem (1)-(2) reads as follows: find $(\mathbf{u}, p) \in(V, M)$ such that for all $(\mathbf{v}, q) \in(V, M)$

$$
\begin{gathered}
a(\mathbf{u}, \mathbf{v}-\mathbf{u})+b(\mathbf{u}, \mathbf{u}, \mathbf{v}-\mathbf{u})+j\left(\mathbf{v}_{\tau}\right)-j\left(\mathbf{u}_{\tau}\right) \\
-d(\mathbf{v}-\mathbf{u}, p) \geq(\mathbf{f}, \mathbf{v}-\mathbf{u}), \\
d(\mathbf{u}, q)=0,
\end{gathered}
$$

where $j(\eta)=\int_{S} g|\eta| d s$ for all $\eta \in L^{2}(S)^{2}$. Saito in [8] showed that there exists some positive $\beta>0$ such that

$$
\beta\|q\| \leq \sup _{v \in V} \frac{d(\mathbf{v}, q)}{\|\mathbf{v}\|_{V}}
$$

then the variational inequality (16) is equivalent to the following: find $\mathbf{u} \in V_{\sigma}$ such that for all $\mathbf{v} \in V_{\sigma}$

$$
a(\mathbf{u}, \mathbf{v}-\mathbf{u})+b(\mathbf{u}, \mathbf{u}, \mathbf{v}-\mathbf{u})+j\left(\mathbf{v}_{\tau}\right)-j\left(\mathbf{u}_{\tau}\right) \geq(\mathbf{f}, \mathbf{v}-\mathbf{u}) .
$$

The existence and uniqueness theorem of the solution $\mathbf{u}$ to the problem (18) has been shown in [19]. Here, we only recall it.

Theorem 1. If the following uniqueness condition holds

$$
\frac{4 \kappa_{1} N\left(\|\mathbf{f}\|+\|g\|_{L^{2}(S)}\right)}{\mu^{2}}<1,
$$

then there exists a unique solution $\mathbf{u} \in V_{\sigma}$ to the variational inequality problem (18) such that

$$
\|\mathbf{u}\|_{V} \leq \frac{2 \kappa_{1}}{\mu}\left(\|\mathbf{f}\|+\|g\|_{L^{2}(S)}\right)<\frac{\mu}{2 N}
$$

where $\kappa_{1}>0$ satisfies

$$
\left|(\mathbf{f}, \mathbf{v})-j\left(\mathbf{v}_{\tau}\right)\right| \leq \kappa_{1}\left(\|\mathbf{f}\|+\|g\|_{L^{2}(S)}\right)\|\mathbf{v}\|_{V}, \quad \forall \mathbf{v} \in V_{\sigma} .
$$

\section{Iteration Penalty Finite Element Approximation}

Suppose that $\Omega$ is a convex and polygon domain. Let $\mathscr{T}_{h}$ be a family of quasi-uniform triangular partition of $\Omega$. The corresponding ordered triangles are denoted by $K_{1}, K_{2}, \ldots, K_{n}$. Let $h_{i}=\operatorname{diam}\left(K_{i}\right), i=1, \ldots, n$, and $h=\max \left\{h_{1}, h_{2}, \ldots, h_{n}\right\}$. For every $K \in \mathscr{T}_{h}$, let $P_{r}(K)$ denote the space of the polynomials on $K$ of degree at most $r$. For simplicity, we consider the conforming finite element spaces $V_{h}$ and $M_{h}$ defined by

$$
\begin{aligned}
& V_{h}=V \cap W_{h} \text { with } W_{h}=\left\{\mathbf{v}_{h} \in C(\bar{\Omega})^{2},\left.\mathbf{v}_{h}\right|_{K}\right. \\
& \left.\in\left[P_{2}(K)\right]^{2}, \forall K \in \mathscr{T}_{h},\right\}, \\
& M_{h}=\left\{q_{h} \in C(\bar{\Omega}),\left.q_{h}\right|_{K} \in P_{1}(K), \forall K \in \mathscr{T}_{h}, \int_{\Omega} q_{h} d x=0\right\} .
\end{aligned}
$$

Denote $V_{0 h}=V_{0} \cap W_{h}$. It is well known that $V_{0 h}$ and $M_{h}$ satisfy the Babuška-Brezzi condition [24, 25]:

$$
\kappa\left\|q_{h}\right\| \leq \sup _{\mathbf{w}_{h} \in V_{0 h}} \frac{d\left(\mathbf{w}_{h}, q_{h}\right)}{\left\|\mathbf{w}_{h}\right\|_{V}},
$$

where $\kappa>0$ is a constant independent of $h$. Denote $R_{h}$ and $Q_{h}$ as the $L^{2}$ orthogonal projections onto $V_{h}$ and $M_{h}$, respectively, which satisfy

$$
\begin{gathered}
\left\|\mathbf{v}-R_{h} \mathbf{v}\right\|+h\left\|\mathbf{v}-R_{h} \mathbf{v}\right\|_{V} \leq c h^{i}\|\mathbf{v}\|_{i}, \\
\forall \mathbf{v} \in H^{3}(\Omega)^{2} \cap V, \quad i=1,2,3, \\
\left\|q-Q_{h} q\right\| \leq c h^{j}\|q\|_{j}, \quad \forall q \in H^{2}(\Omega) \cap M, \quad j=1,2 .
\end{gathered}
$$

It follows from the trace inequality $\|\mathbf{v}\|_{L^{2}(S)} \leq c\|\mathbf{v}\|^{1 / 2}\|\mathbf{v}\|_{V}^{1 / 2}$ [26] that

$$
\begin{aligned}
\| \mathbf{v}- & R_{h} \mathbf{v} \|_{L^{2}(S)} \\
& \leq c\left\|\mathbf{v}-R_{h} \mathbf{v}\right\|^{1 / 2}\left\|\mathbf{v}-R_{h} \mathbf{v}\right\|_{V}^{1 / 2} \\
& \leq c h^{i-1 / 2}\|\mathbf{v}\|_{i}, \quad \forall \mathbf{v} \in H^{3}(\Omega)^{2} \cap V, \quad i=1,2,3 .
\end{aligned}
$$

Let $\varepsilon>0$ be some small parameter. The one-level iteration penalty finite element method for the problem (16) has been studied in [20], which can be described as follows.

Step 1. Find $\left(\mathbf{u}_{\varepsilon h}^{0}, p_{\varepsilon h}^{0}\right) \in\left(V_{h}, M_{h}\right)$ such that for all $\left(\mathbf{v}_{h}, q_{h}\right) \in$ $\left(V_{h}, M_{h}\right)$

$$
\begin{gathered}
a\left(\mathbf{u}_{\varepsilon h}^{0}, \mathbf{v}_{h}-\mathbf{u}_{\varepsilon h}^{0}\right)+b\left(\mathbf{u}_{\varepsilon h}^{0}, \mathbf{u}_{\varepsilon h}^{0}, \mathbf{v}_{h}-\mathbf{u}_{\varepsilon h}^{0}\right)+j\left(\mathbf{v}_{h \tau}\right) \\
-j\left(\mathbf{u}_{\varepsilon h \tau}^{0}\right)-d\left(\mathbf{v}_{h}-\mathbf{u}_{\varepsilon h}^{0}, p_{\varepsilon h}^{0}\right) \geq\left(\mathbf{f}, \mathbf{v}_{h}-\mathbf{u}_{\varepsilon h}^{0}\right), \\
d\left(\mathbf{u}_{\varepsilon h}^{0}, q_{h}\right)+\varepsilon\left(p_{\varepsilon h}^{0}, q_{h}\right)=0 .
\end{gathered}
$$

Step 2. For $k=1,2, \ldots$, find $\left(\mathbf{u}_{\varepsilon h}^{k}, p_{\varepsilon h}^{k}\right) \in\left(V_{h}, M_{h}\right)$ such that for all $\left(\mathbf{v}_{h}, q_{h}\right) \in\left(V_{h}, M_{h}\right)$

$$
\begin{gathered}
a\left(\mathbf{u}_{\varepsilon h}^{k}, \mathbf{v}_{h}-\mathbf{u}_{\varepsilon h}^{k}\right)+b\left(\mathbf{u}_{\varepsilon h}^{k}, \mathbf{u}_{\varepsilon h}^{k}, \mathbf{v}_{h}-\mathbf{u}_{\varepsilon h}^{k}\right)+j\left(\mathbf{v}_{h \tau}\right) \\
-j\left(\mathbf{u}_{\varepsilon h \tau}^{k}\right)-d\left(\mathbf{v}_{h}-\mathbf{u}_{\varepsilon h}^{k}, p_{\varepsilon h}^{k}\right) \geq\left(\mathbf{f}, \mathbf{v}_{h}-\mathbf{u}_{\varepsilon h}^{k}\right), \\
d\left(\mathbf{u}_{\varepsilon h}^{k}, q_{h}\right)+\varepsilon\left(p_{\varepsilon h}^{k}, q_{h}\right)=\varepsilon\left(p_{\varepsilon h}^{k-1}, q_{h}\right) .
\end{gathered}
$$

First, we give the a priori estimate of the solution $\left(\mathbf{u}_{\varepsilon h}^{k}, p_{\varepsilon h}^{k}\right)$ to the problem (28). 
Theorem 2. Suppose that $\left(\mathbf{u}_{\varepsilon h}^{k}, p_{\varepsilon h}^{k}\right) \in\left(V_{h}, M_{h}\right)$ is the solution to the problem (28); then it satisfies

$$
\mu\left\|\mathbf{u}_{\varepsilon h}^{k}\right\|_{V}^{2}+\varepsilon\left\|p_{\varepsilon h}^{k}\right\|^{2} \leq \frac{(2 k+1) \kappa_{1}^{2}}{2 \mu}\left(\|\mathbf{f}\|+\|g\|_{L^{2}(S)}\right)^{2} .
$$

Proof. Setting $\mathbf{v}_{h}=0, q_{h}=p_{\varepsilon h}^{0}$ in (27), using (12) and Young's inequality, it yields that

$$
\begin{aligned}
\mu\left\|\mathbf{u}_{\varepsilon h}^{0}\right\|_{V}^{2}+\varepsilon\left\|p_{\varepsilon h}^{0}\right\|^{2} \\
\quad \leq\left(\mathbf{f}, \mathbf{u}_{\varepsilon h}^{0}\right)-j\left(\mathbf{u}_{\varepsilon h \tau}^{0}\right) \\
\leq \kappa_{1}\left(\|\mathbf{f}\|+\|g\|_{L^{2}(S)}\right)\left\|\mathbf{u}_{\varepsilon h}^{0}\right\|_{V} \\
\quad \leq \frac{\mu}{2}\left\|\mathbf{u}_{\varepsilon h}^{0}\right\|_{V}^{2}+\frac{\kappa_{1}^{2}}{2 \mu}\left(\|\mathbf{f}\|+\|g\|_{L^{2}(S)}\right)^{2} .
\end{aligned}
$$

Then we have

$$
\mu\left\|\mathbf{u}_{\varepsilon h}^{0}\right\|_{V}^{2}+2 \varepsilon\left\|p_{\varepsilon h}^{0}\right\|^{2} \leq \frac{\kappa_{1}^{2}}{\mu}\left(\|\mathbf{f}\|+\|g\|_{L^{2}(S)}\right)^{2} .
$$

For $k=1,2, \ldots$, setting $\mathbf{v}_{h}=0, q_{h}=p_{\varepsilon h}^{k}$ in (28), it yields that

$$
\begin{aligned}
\mu\left\|\mathbf{u}_{\varepsilon h}^{k}\right\|_{V}^{2}+\varepsilon\left\|p_{\varepsilon h}^{k}\right\|^{2} \\
\leq\left(\mathbf{f}, \mathbf{u}_{\varepsilon h}^{k}\right)-j\left(\mathbf{u}_{\varepsilon h \tau}^{k}\right)+\varepsilon\left(p_{\varepsilon h}^{k-1}, p_{\varepsilon h}^{k}\right) \\
\leq \frac{\mu}{2}\left\|\mathbf{u}_{\varepsilon h}^{k}\right\|_{V}^{2}+\frac{\kappa_{1}^{2}}{2 \mu}\left(\|\mathbf{f}\|+\|g\|_{L^{2}(S)}\right)^{2} \\
+\frac{\varepsilon}{2}\left\|p_{\varepsilon h}^{k}\right\|^{2}+\frac{\varepsilon}{2}\left\|p_{\varepsilon h}^{k-1}\right\|^{2} .
\end{aligned}
$$

Thus, we obtain

$$
\begin{aligned}
\mu\left\|\mathbf{u}_{\varepsilon h}^{k}\right\|_{V}^{2}+\varepsilon\left\|p_{\varepsilon h}^{k}\right\|^{2} \\
\quad \leq \frac{\kappa_{1}^{2}}{\mu}\left(\|\mathbf{f}\|+\|g\|_{L^{2}(S)}\right)^{2}+\varepsilon\left\|p_{\varepsilon h}^{k-1}\right\|^{2} \\
\leq \cdots \leq \frac{k \kappa_{1}^{2}}{\mu}\left(\|\mathbf{f}\|+\|g\|_{L^{2}(S)}\right)^{2}+\varepsilon\left\|p_{\varepsilon h}^{0}\right\|^{2} \\
\leq \frac{(2 k+1) \kappa_{1}^{2}}{2 \mu}\left(\|\mathbf{f}\|+\|g\|_{L^{2}(S)}\right)^{2} .
\end{aligned}
$$

The next theorem gives the error estimate between the solutions (u, $p)$ and $\left(\mathbf{u}_{\varepsilon h}^{k}, p_{\varepsilon h}^{k}\right)$ to the problems (16) and (28), respectively. The proof can be found in [20].

Theorem 3. Let $(\mathbf{u}, p) \in H^{3}(\Omega)^{2} \cap V \times H^{2}(\Omega) \cap M$ and $\left(\mathbf{u}_{\varepsilon h}^{k}, p_{\varepsilon h}^{k}\right) \in\left(V_{h}, M_{h}\right)$ be the solutions to the problems (16) and (28), respectively; then they satisfy

$$
\left\|\mathbf{u}-\mathbf{u}_{\varepsilon h}^{k}\right\|_{V}+\left\|p-p_{\varepsilon h}^{k}\right\| \leq c\left(h^{5 / 4}+\varepsilon^{k+1}\right) .
$$

Next, we will show the error estimate $\left\|\mathbf{u}-\mathbf{u}_{\varepsilon h}^{k}\right\|$ for the penalty finite element approximation (28). This $L^{2}$ error analysis is based on the regularity assumption that the following linearized problem $(35)$ is $\left(H^{2}(\Omega)^{2}, H^{1}(\Omega)\right)$ regular.

Given $\mathbf{z} \in L^{2}(\Omega)^{2}$, find $(\mathbf{w}, \pi) \in(V, M)$ such that for all $(\mathbf{v}, q) \in(V, M)$

$$
\begin{gathered}
a(\mathbf{w}, \mathbf{v})+b\left(\mathbf{u}_{\varepsilon h}^{k}, \mathbf{v}, \mathbf{w}\right)+b(\mathbf{v}, \mathbf{u}, \mathbf{w})-d(\mathbf{v}, \pi)=(\mathbf{z}, \mathbf{v}), \\
d(\mathbf{w}, q)=0 .
\end{gathered}
$$

According to (12) and (20), it is easy to verify that there exists a unique solution $(\mathbf{w}, \pi)$ to the problem (35). The assumption that $(35)$ is $\left(H^{2}(\Omega)^{2}, H^{1}(\Omega)\right)$ regular means that $(\mathbf{w}, \pi)$ also belongs to $\left(H^{2}(\Omega)^{2}, H^{1}(\Omega)\right)$ and the following inequality holds:

$$
\|\mathbf{w}\|_{2}+\|\pi\|_{1} \leq c\|z\| \text {. }
$$

Let $I_{h}$ be the $L^{2}$ orthogonal projections onto $V_{0 h}$ and satisfy

$$
\left\|\mathbf{w}-I_{h} \mathbf{w}\right\|_{V} \leq c h\|\mathbf{w}\|_{2} \text {. }
$$

Theorem 4. Let $(\mathbf{u}, p) \in H^{3}(\Omega)^{2} \cap V \times H^{2}(\Omega) \cap M$ and $\left(\mathbf{u}_{\varepsilon h}^{k}, p_{\varepsilon h}^{k}\right) \in\left(V_{h}, M_{h}\right)$ be the solutions to the problems (16) and (28), respectively; then they satisfy

$$
\left\|\mathbf{u}-\mathbf{u}_{\varepsilon h}^{k}\right\| \leq c\left(h^{9 / 4}+\varepsilon h^{5 / 4}+\varepsilon^{k+1}\right) .
$$

Proof. Setting $\mathbf{z}=\mathbf{u}-\mathbf{u}_{\varepsilon h}^{k}$ and $\mathbf{v}=\mathbf{u}-\mathbf{u}_{\varepsilon h}^{k}$ in the first equation of (35), we get

$$
\begin{aligned}
\left\|\mathbf{u}-\mathbf{u}_{\varepsilon h}^{k}\right\|^{2}= & a\left(\mathbf{w}, \mathbf{u}-\mathbf{u}_{\varepsilon h}^{k}\right)+b\left(\mathbf{u}_{\varepsilon h}^{k}, \mathbf{u}-\mathbf{u}_{\varepsilon h}^{k}, w\right) \\
& +b\left(\mathbf{u}-\mathbf{u}_{\varepsilon h}^{k}, \mathbf{u}, \mathbf{w}\right)-d\left(\mathbf{u}-\mathbf{u}_{\varepsilon h}^{k}, \pi\right) .
\end{aligned}
$$

Taking $\mathbf{v}=\mathbf{u} \pm I_{h} \mathbf{w}, q=Q_{h} \pi$ in (16) and $\mathbf{v}_{h}=\mathbf{u}_{\varepsilon h}^{k} \pm I_{h} \mathbf{w}, q_{h}=$ $Q_{h} \pi$ in (28), respectively, we obtain

$$
\begin{gathered}
a\left(\mathbf{u}, I_{h} \mathbf{w}\right)+b\left(\mathbf{u}, \mathbf{u}, I_{h} \mathbf{w}\right)-d\left(I_{h} \mathbf{w}, p\right)=\left(\mathbf{f}, I_{h} \mathbf{w}\right), \\
d\left(\mathbf{u}, Q_{h} \pi\right)=0, \\
a\left(\mathbf{u}_{\varepsilon h}^{k}, I_{h} \mathbf{w}\right)+b\left(\mathbf{u}_{\varepsilon h}^{k}, \mathbf{u}_{\varepsilon h}^{k}, I_{h} \mathbf{w}\right)-d\left(I_{h} \mathbf{w}, p_{\varepsilon h}^{k}\right)=\left(\mathbf{f}, I_{h} \mathbf{w}\right), \\
d\left(\mathbf{u}_{\varepsilon h}^{k}, Q_{h} \pi\right)+\varepsilon\left(p_{\varepsilon h}^{k}, Q_{h} \pi\right)=\varepsilon\left(p_{\varepsilon h}^{k-1}, Q_{h} \pi\right) .
\end{gathered}
$$

Subtracting them, we get

$$
\begin{gathered}
a\left(\mathbf{u}-\mathbf{u}_{\varepsilon h}^{k}, I_{h} \mathbf{w}\right)+b\left(\mathbf{u}, \mathbf{u}, I_{h} \mathbf{w}\right)-b\left(\mathbf{u}_{\varepsilon h}^{k}, \mathbf{u}_{\varepsilon h}^{k}, I_{h} \mathbf{w}\right) \\
\quad-d\left(I_{h} \mathbf{w}, p-p_{\varepsilon h}^{k}\right)=0, \\
d\left(\mathbf{u}-\mathbf{u}_{\varepsilon h}^{k}, Q_{h} \pi\right)+\varepsilon\left(p_{\varepsilon h}^{k-1}, Q_{h} \pi\right)-\varepsilon\left(p_{\varepsilon h}^{k}, Q_{h} \pi\right)=0 .
\end{gathered}
$$


Substituting the previous equation into (39), it yields that

$$
\begin{aligned}
\left\|\mathbf{u}-\mathbf{u}_{\varepsilon h}^{k}\right\|^{2} & =\underbrace{a\left(\mathbf{w}-I_{h} \mathbf{w}, \mathbf{u}-\mathbf{u}_{\varepsilon h}^{k}\right)}_{J_{1}}+\underbrace{b\left(\mathbf{u}_{\varepsilon h}^{k}, \mathbf{u}-\mathbf{u}_{\varepsilon h}^{k}, \mathbf{w}\right)}_{J_{2}} \\
& \underbrace{+b\left(\mathbf{u}-\mathbf{u}_{\varepsilon h}^{k}, \mathbf{u}, \mathbf{w}\right)+b\left(\mathbf{u}_{\varepsilon h}^{k}, \mathbf{u}_{\varepsilon h}^{k}, I_{h} \mathbf{w}\right)-b\left(\mathbf{u}, \mathbf{u}, I_{h} \mathbf{w}\right)}_{J_{2}} \\
& +\underbrace{d\left(I_{h} \mathbf{w}-\mathbf{w}, p-p_{\varepsilon h}^{k}\right)-d\left(\mathbf{u}-\mathbf{u}_{\varepsilon h}^{k}, \pi-Q_{h} \pi\right)}_{J_{3}} \\
& +\underbrace{\varepsilon\left(p_{\varepsilon h}^{k-1}, Q_{h} \pi\right)-\varepsilon\left(p_{\varepsilon h}^{k}, Q_{h} \pi\right) .}_{J_{4}}
\end{aligned}
$$

Using (34), (36), and (37), $J_{1}$ is estimated by

$$
\begin{aligned}
J_{1} & =a\left(\mathbf{w}-I_{h} \mathbf{w}, \mathbf{u}-\mathbf{u}_{\varepsilon h}^{k}\right) \\
& \leq \mu\left\|\mathbf{u}-\mathbf{u}_{\varepsilon h}^{k}\right\|_{V}\left\|\mathbf{w}-I_{h} \mathbf{w}\right\|_{V} \\
& \leq \operatorname{ch}\left(h^{5 / 4}+\varepsilon^{k+1}\right)\|\mathbf{w}\|_{2} \\
& \leq \operatorname{ch}\left(h^{5 / 4}+\varepsilon^{k+1}\right)\left\|\mathbf{u}-\mathbf{u}_{\varepsilon h}^{k}\right\| .
\end{aligned}
$$

Similarly, using (25), (34), (36), and (37), $J_{3}$ is estimated by

$$
\begin{aligned}
J_{3} & =d\left(I_{h} \mathbf{w}-\mathbf{w}, p-p_{\varepsilon h}^{k}\right)-d\left(\mathbf{u}-\mathbf{u}_{\varepsilon h}^{k}, \pi-Q_{h} \pi\right) \\
& \leq\left\|I_{h} \mathbf{w}-\mathbf{w}\right\|_{V}\left\|p-p_{\varepsilon h}^{k}\right\|+\left\|\mathbf{u}-\mathbf{u}_{\varepsilon h}^{k}\right\|_{V}\left\|\pi-Q_{h} \pi\right\| \\
& \leq \operatorname{ch}\left(h^{5 / 4}+\varepsilon^{k+1}\right)\left(\|\mathbf{w}\|_{2}+\|\pi\|_{1}\right) \\
& \leq \operatorname{ch}\left(h^{5 / 4}+\varepsilon^{k+1}\right)\left\|\mathbf{u}-\mathbf{u}_{\varepsilon h}^{k}\right\| .
\end{aligned}
$$

We rewrite $J_{2}$ as

$$
\begin{aligned}
J_{2}= & b\left(\mathbf{u}_{\varepsilon h}^{k}, \mathbf{u}-\mathbf{u}_{\varepsilon h}^{k}, w\right)+b\left(\mathbf{u}-\mathbf{u}_{\varepsilon h}^{k}, \mathbf{u}, \mathbf{w}\right) \\
& +b\left(\mathbf{u}_{\varepsilon h}^{k}, \mathbf{u}_{\varepsilon h}^{k}, I_{h} \mathbf{w}\right)-b\left(\mathbf{u}, \mathbf{u}, I_{h} \mathbf{w}\right) \\
= & b\left(\mathbf{u}_{\varepsilon h}^{k}, \mathbf{u}-\mathbf{u}_{\varepsilon h}^{k}, \mathbf{w}\right)+b\left(\mathbf{u}-\mathbf{u}_{\varepsilon h}^{k}, \mathbf{u}, \mathbf{w}\right) \\
& +b\left(\mathbf{u}_{\varepsilon h}^{k}, \mathbf{u}_{\varepsilon h}^{k}-\mathbf{u}, I_{h} \mathbf{w}\right)-b\left(\mathbf{u}-\mathbf{u}_{\varepsilon h}^{k}, \mathbf{u}, I_{h} \mathbf{w}\right) \\
= & b\left(\mathbf{u}_{\varepsilon h}^{k}, \mathbf{u}-\mathbf{u}_{\varepsilon h}^{k}, \mathbf{w}-I_{h} \mathbf{w}\right)+b\left(\mathbf{u}-\mathbf{u}_{\varepsilon h}^{k}, \mathbf{u}, \mathbf{w}-I_{h} \mathbf{w}\right) .
\end{aligned}
$$

Then, from (13), (20), (29), (34), (36), and (37), it holds that

$$
\begin{aligned}
J_{2} & \leq N\left(\left\|\mathbf{u}_{\varepsilon h}^{k}\right\|_{V}+\|\mathbf{u}\|_{V}\right)\left\|\mathbf{u}-\mathbf{u}_{\varepsilon h}^{k}\right\|_{V}\left\|\mathbf{w}-R_{h} \mathbf{w}\right\|_{V} \\
& \leq \operatorname{ch}\left(h^{5 / 4}+\varepsilon^{k+1}\right)\|\mathbf{w}\|_{2} \\
& \leq \operatorname{ch}\left(h^{5 / 4}+\varepsilon^{k+1}\right)\left\|\mathbf{u}-\mathbf{u}_{\varepsilon h}^{k}\right\| .
\end{aligned}
$$

Finally, we estimate $J_{4}$ by

$$
\begin{aligned}
J_{4} & =\varepsilon\left(p_{\varepsilon h}^{k-1}, Q_{h} \pi\right)-\varepsilon\left(p_{\varepsilon h}^{k}, Q_{h} \pi\right) \\
& =\varepsilon\left(p_{\varepsilon h}^{k-1}-p, Q_{h} \pi\right)+\varepsilon\left(p-p_{\varepsilon h}^{k}, Q_{h} \pi\right) \\
& \leq \varepsilon\left(\left\|p_{\varepsilon h}^{k-1}-p\right\|+\left\|p-p_{\varepsilon h}^{k}\right\|\right)\left\|Q_{h} \pi\right\| \\
& \leq c \varepsilon\left(h^{5 / 4}+\varepsilon^{k}\right)\|\pi\|_{1} \\
& \leq c \varepsilon\left(h^{5 / 4}+\varepsilon^{k}\right)\left\|\mathbf{u}-\mathbf{u}_{\varepsilon h}^{k}\right\| .
\end{aligned}
$$

Combining these estimates with (42), we conclude that (38) holds.

\section{Two-Level Iteration Penalty Methods}

In this section, based on the iteration penalty method described in the previous section, the two-level iteration penalty finite element methods for (16) are proposed in terms of the Stokes iteration, Oseen iteration, or Newtonian iteration. From now on, $H$ and $h$ with $h<H$ are two real positive parameters. The coarse mesh triangulation $\mathscr{T}_{H}$ is made as in Section 3. And a fine mesh triangulation $\mathscr{T}_{h}$ is generated by a mesh refinement process to $\mathscr{T}_{H}$. The conforming finite element space pairs $\left(V_{h}, M_{h}\right)$ and $\left(V_{H}, M_{H}\right) \subset\left(V_{h}, M_{h}\right)$ corresponding to the triangulations $\mathscr{T}_{h}$ and $\mathscr{T}_{H}$, respectively, are constructed as in Section 3. With the preavious notations, we propose the following two-level iteration finite element methods.

4.1. Two-Level Stokes Iteration Penalty Method. In Steps 1 and 2, we solve (27) and (28) on the coarse mesh, as in the follwing.

Step 1. Find $\left(\mathbf{u}_{\varepsilon H}^{0}, p_{\varepsilon H}^{0}\right) \in\left(V_{H}, M_{H}\right)$ such that for all $\left(\mathbf{v}_{H}, q_{H}\right) \in\left(V_{H}, M_{H}\right)$

$$
\begin{gathered}
a\left(\mathbf{u}_{\varepsilon H}^{0}, \mathbf{v}_{H}-\mathbf{u}_{\varepsilon H}^{0}\right)+b\left(\mathbf{u}_{\varepsilon H}^{0}, \mathbf{u}_{\varepsilon H}^{0}, \mathbf{v}_{H}-\mathbf{u}_{\varepsilon H}^{0}\right)+j\left(\mathbf{v}_{H \tau}\right) \\
-j\left(\mathbf{u}_{\varepsilon H \tau}^{0}\right)-d\left(\mathbf{v}_{H}-\mathbf{u}_{\varepsilon H}^{0}, p_{\varepsilon H}^{0}\right) \geq\left(\mathbf{f}, \mathbf{v}_{H}-\mathbf{u}_{\varepsilon H}^{0}\right), \\
d\left(\mathbf{u}_{\varepsilon H}^{0}, q_{H}\right)+\varepsilon\left(p_{\varepsilon H}^{0}, q_{H}\right)=0 .
\end{gathered}
$$

Step 2. For $k=1,2, \ldots$, find $\left(\mathbf{u}_{\varepsilon H}^{k}, p_{\varepsilon H}^{k}\right) \in\left(V_{H}, M_{H}\right)$ such that for all $\left(\mathbf{v}_{H}, q_{H}\right) \in\left(V_{H}, M_{H}\right)$

$$
\begin{gathered}
a\left(\mathbf{u}_{\varepsilon H}^{k}, \mathbf{v}_{H}-\mathbf{u}_{\varepsilon H}^{k}\right)+b\left(\mathbf{u}_{\varepsilon H}^{k}, \mathbf{u}_{\varepsilon H}^{k}, \mathbf{v}_{H}-\mathbf{u}_{\varepsilon H}^{k}\right)+j\left(\mathbf{v}_{H \tau}\right) \\
-j\left(\mathbf{u}_{\varepsilon H \tau}^{k}\right)-d\left(\mathbf{v}_{H}-\mathbf{u}_{\varepsilon H}^{k}, p_{\varepsilon H}^{k}\right) \geq\left(\mathbf{f}, \mathbf{v}_{H}-\mathbf{u}_{\varepsilon H}^{k}\right), \\
d\left(\mathbf{u}_{\varepsilon H}^{k}, q_{H}\right)+\varepsilon\left(p_{\varepsilon H}^{k}, q_{H}\right)=\varepsilon\left(p_{\varepsilon H}^{k-1}, q_{h}\right) .
\end{gathered}
$$

In Step 3, we solve a Stokes-type variational inequality problem on the fine mesh in terms of the Stokes iteration, as in the following. 
Step 3. Find $\left(\mathbf{u}_{\varepsilon h}, p_{\varepsilon h}\right) \in\left(V_{h}, M_{h}\right)$ such that for all $\left(\mathbf{v}_{h}, q_{h}\right) \in$ $\left(V_{h}, M_{h}\right)$

$$
\begin{gathered}
a\left(\mathbf{u}_{\varepsilon h}, \mathbf{v}_{h}-\mathbf{u}_{\varepsilon h}\right)+b\left(\mathbf{u}_{\varepsilon H}^{k}, \mathbf{u}_{\varepsilon H}^{k}, \mathbf{v}_{h}-\mathbf{u}_{\varepsilon h}\right)+j\left(\mathbf{v}_{h \tau}\right) \\
-j\left(\mathbf{u}_{\varepsilon h \tau}\right)-d\left(\mathbf{v}_{h}-\mathbf{u}_{\varepsilon h}, p_{\varepsilon h}\right) \geq\left(\mathbf{f}, \mathbf{v}_{h}-\mathbf{u}_{\varepsilon h}\right), \\
d\left(\mathbf{u}_{\varepsilon h}, q_{h}\right)+\varepsilon\left(p_{\varepsilon h}, q_{h}\right)=\varepsilon\left(p_{\varepsilon H}^{k}, q_{h}\right) .
\end{gathered}
$$

As a direct consequence of Theorem 2, the solution $\left(\mathbf{u}_{\varepsilon H}^{k}, p_{\varepsilon H}^{k}\right)$ to the problem (49) satisfies

$$
\begin{aligned}
& \left\|\mathbf{u}_{\varepsilon H}^{k}\right\|_{V} \leq \sqrt{\frac{2 k+1}{2}} \frac{\kappa_{1}}{\mu}\left(\|\mathbf{f}\|+\|g\|_{L^{2}(S)}\right), \\
& \varepsilon\left\|p_{\varepsilon H}^{k}\right\|^{2} \leq \frac{(2 k+1) \kappa_{1}^{2}}{2 \mu}\left(\|\mathbf{f}\|+\|g\|_{L^{2}(S)}\right)^{2} .
\end{aligned}
$$

Next, we estimate $\mathbf{u}_{\varepsilon h}$. Taking $\mathbf{v}_{h}=0, q_{h}=p_{\varepsilon h}$ in (50), it yields

$$
\begin{aligned}
& \mu\left\|\mathbf{u}_{\varepsilon h}\right\|_{V}^{2}+\varepsilon\left\|p_{\varepsilon h}\right\|^{2} \\
& \leq\left(\mathbf{f}, \mathbf{u}_{\varepsilon h}\right)-j\left(\mathbf{u}_{\varepsilon h \tau}\right)-b\left(\mathbf{u}_{\varepsilon H}^{k}, \mathbf{u}_{\varepsilon H}^{k}, \mathbf{u}_{\varepsilon h}\right)+\varepsilon\left(p_{\varepsilon H}^{k}, p_{\varepsilon h}\right) \\
& \leq \kappa_{1}\left(\|\mathbf{f}\|+\|g\|_{L^{2}(S)}\right)\left\|\mathbf{u}_{\varepsilon h}\right\|_{V} \\
&+N\left\|\mathbf{u}_{\varepsilon H}^{k}\right\|_{V}^{2}\left\|\mathbf{u}_{\varepsilon h}\right\|_{V}+\varepsilon\left\|p_{\varepsilon H}^{k}\right\|\left\|p_{\varepsilon h}\right\| \\
& \leq \frac{\mu}{2}\left\|\mathbf{u}_{\varepsilon h}\right\|_{V}^{2}+\frac{\varepsilon}{2}\left\|p_{\varepsilon h}\right\|^{2}+\frac{\varepsilon}{2}\left\|p_{\varepsilon H}^{k}\right\|^{2} \\
&+\frac{\kappa_{1}^{2}}{\mu}\left(\|\mathbf{f}\|+\|g\|_{L^{2}(S)}\right)^{2}+\frac{N^{2}}{\mu}\left\|\mathbf{u}_{\varepsilon H}^{k}\right\|_{V^{*}}^{4}
\end{aligned}
$$

That is,

$$
\left\|\mathbf{u}_{\varepsilon h}\right\|_{V} \leq \frac{\sqrt{2} \kappa_{1}}{\mu}\left(\|\mathbf{f}\|+\|g\|_{L^{2}(S)}\right)+\frac{\sqrt{2} N}{\mu}\left\|\mathbf{u}_{\varepsilon H}^{k}\right\|_{V}^{2}+\frac{\sqrt{\varepsilon}}{\sqrt{\mu}}\left\|p_{\varepsilon H}^{k}\right\| .
$$

Suppose that the initial data satisfies

$$
\frac{7 N \kappa_{1}}{\mu^{2}} \sqrt{\frac{2 k+1}{2}}\left(\|\mathbf{f}\|+\|g\|_{L^{2}(S)}\right)<1 ;
$$

then using (51)-(52), we can estimate $u_{\varepsilon h}$ by

$$
\begin{aligned}
\left\|\mathbf{u}_{\varepsilon h}\right\|_{V} & \leq \frac{\sqrt{2} \kappa_{1}}{\mu}\left(\|\mathbf{f}\|+\|g\|_{L^{2}(S)}\right)+\frac{\sqrt{2}}{7}\left\|\mathbf{u}_{\varepsilon H}^{k}\right\|_{V}+\frac{\mu}{7 N} \\
& \leq\left(\sqrt{2}+\frac{\sqrt{2 k+1}}{7}\right) \frac{\kappa_{1}}{\mu}\left(\|\mathbf{f}\|+\|g\|_{L^{2}(S)}\right)+\frac{\mu}{12 N} \\
& \leq\left(\sqrt{2}+\frac{\sqrt{2 k+1}}{7}\right) \frac{\sqrt{2} \mu}{7 \sqrt{2 k+1} N}+\frac{\mu}{12 N} \\
& <\left(\frac{2}{7}+\frac{2}{49}+\frac{1}{7}\right) \frac{\mu}{N}=\frac{23 \mu}{49 N}<\frac{\mu}{2 N} .
\end{aligned}
$$

By the classical existence theorem for the variational inequality problem of the second kind in the finite dimension [27], we have the following.

Theorem 5. Under the uniqueness condition (55), there exists a unique solution $\left(\mathbf{u}_{\varepsilon h}, p_{\varepsilon h}\right)$ to the problem (50). Moreover, $\mathbf{u}_{\varepsilon h}$ satisfy (56).

It follows from Theorems 3 and 4 that $\left(\mathbf{u}_{\varepsilon H}^{k}, p_{\varepsilon H}^{k}\right)$ is of the following error estimates:

$$
\begin{gathered}
\left\|\mathbf{u}-\mathbf{u}_{\varepsilon H}^{k}\right\|_{V}+\left\|p-p_{\varepsilon H}^{k}\right\| \leq c\left(H^{5 / 4}+\varepsilon^{k+1}\right), \\
\left\|\mathbf{u}-\mathbf{u}_{\varepsilon H}^{k}\right\| \leq c\left(H^{9 / 4}+\varepsilon H^{5 / 4}+\varepsilon^{k+1}\right) .
\end{gathered}
$$

Next, we begin to prove the following error estimate for the solution $\left(\mathbf{u}_{\varepsilon h}, p_{\varepsilon h}\right)$ to the problem (50).

Theorem 6. Suppose that the uniqueness condition (55) holds. Let $(\mathbf{u}, p) \in H^{3}(\Omega)^{2} \cap V \times H^{2}(\Omega) \cap M$ and $\left(\mathbf{u}_{\varepsilon h}, p_{\varepsilon h}\right) \in$ $\left(V_{h}, M_{h}\right)$ be the solutions to the problems (16) and (50), respectively; then they satisfy

$$
\left\|\mathbf{u}-\mathbf{u}_{\varepsilon h}\right\|_{V}+\left\|p-p_{\varepsilon h}\right\| \leq c\left(h^{5 / 4}+\varepsilon H^{5 / 4}+H^{9 / 4}+\varepsilon^{k+1}\right) .
$$

Proof. Define a generalized bilinear form on $\left(V_{h}, M_{h}\right) \times$ $\left(V_{h}, M_{h}\right)$ by

$$
\begin{aligned}
\mathscr{B}_{\varepsilon h}\left(\mathbf{u}_{h}, p_{h} ; \mathbf{v}_{h}, q_{h}\right)= & a\left(\mathbf{u}_{h}, \mathbf{v}_{h}\right)-d\left(\mathbf{v}_{h}, p_{h}\right) \\
& +d\left(\mathbf{u}_{h}, q_{h}\right)+\varepsilon\left(p_{h}, q_{h}\right) .
\end{aligned}
$$

Taking $\mathbf{v}_{h}=R_{h} \mathbf{u}, q_{h}=p_{\varepsilon h}-Q_{h} p$ in (50), we have

$$
\begin{aligned}
\mu \| \mathbf{u}_{\varepsilon h}- & R_{h} \mathbf{u}\left\|_{V}^{2}+\varepsilon\right\| p_{\varepsilon h}-Q_{h} p \|^{2} \\
= & \mathscr{B}_{\varepsilon h}\left(\mathbf{u}_{\varepsilon h}-R_{h} \mathbf{u}, p_{\varepsilon h}-Q_{h} p ; \mathbf{u}_{\varepsilon h}-R_{h} \mathbf{u}, p_{\varepsilon h}-Q_{h} p\right) \\
= & a\left(\mathbf{u}_{\varepsilon h}, \mathbf{u}_{\varepsilon h}-R_{h} \mathbf{u}\right)-d\left(\mathbf{u}_{\varepsilon h}-R_{h} \mathbf{u}, p_{\varepsilon h}\right) \\
& +d\left(\mathbf{u}_{\varepsilon h}, p_{\varepsilon h}-Q_{h} p\right)+\varepsilon\left(p_{\varepsilon h}, p_{\varepsilon h}-Q_{h} p\right) \\
& -\mathscr{B}_{\varepsilon h}\left(R_{h} \mathbf{u}, Q_{h} p ; \mathbf{u}_{\varepsilon h}-R_{h} \mathbf{u}, p_{\varepsilon h}-Q_{h} p\right) \\
\leq & \left(\mathbf{f}, \mathbf{u}_{\varepsilon h}-R_{h} \mathbf{u}\right)+b\left(\mathbf{u}_{\varepsilon H}^{k}, \mathbf{u}_{\varepsilon H}^{k}, R_{h} \mathbf{u}-\mathbf{u}_{\varepsilon h}\right) \\
& +\varepsilon\left(p_{\varepsilon H}^{k}, p_{\varepsilon h}-Q_{h} p\right)+j\left(R_{h} \mathbf{u}_{\tau}\right)-j\left(\mathbf{u}_{\varepsilon h \tau}\right) \\
& -\mathscr{B}_{\varepsilon h}\left(R_{h} \mathbf{u}, Q_{h} p ; \mathbf{u}_{\varepsilon h}-R_{h} \mathbf{u}, p_{\varepsilon h}-Q_{h} p\right) .
\end{aligned}
$$

Let $\mathbf{v}=\mathbf{u}_{\varepsilon h}$ and $\mathbf{v}=2 \mathbf{u}-R_{h} \mathbf{u}$ in the first inequality of (16); then

$$
\begin{gathered}
a\left(\mathbf{u}, \mathbf{u}_{\varepsilon h}-\mathbf{u}\right)+b\left(\mathbf{u}, \mathbf{u}, \mathbf{u}_{\varepsilon h}-u\right)+j\left(\mathbf{u}_{\varepsilon h \tau}\right) \\
\quad-j\left(\mathbf{u}_{\tau}\right)-d\left(\mathbf{u}_{\varepsilon h}-\mathbf{u}, p\right) \geq\left(\mathbf{f}, \mathbf{u}_{\varepsilon h}-\mathbf{u}\right), \\
a\left(\mathbf{u}, \mathbf{u}-R_{h} \mathbf{u}\right)+b\left(\mathbf{u}, \mathbf{u}, \mathbf{u}-R_{h} \mathbf{u}\right)+j\left(2 \mathbf{u}_{\tau}-R_{h} \mathbf{u}_{\tau}\right) \\
\quad-j\left(\mathbf{u}_{\tau}\right)-d\left(\mathbf{u}-R_{h} \mathbf{u}, p\right) \geq\left(\mathbf{f}, \mathbf{u}-R_{h} \mathbf{u}\right) .
\end{gathered}
$$


Adding the above two inequalities gives

$$
\begin{aligned}
\left(\mathbf{f}, \mathbf{u}_{\varepsilon h}-R_{h} \mathbf{u}\right) \leq a\left(\mathbf{u}, \mathbf{u}_{\varepsilon h}-R_{h} \mathbf{u}\right) \\
\quad+b\left(\mathbf{u}, \mathbf{u}, \mathbf{u}_{\varepsilon h}-R_{h} \mathbf{u}\right)-d\left(\mathbf{u}_{\varepsilon h}-R_{h} \mathbf{u}, p\right) \\
\quad+j\left(2 \mathbf{u}_{\tau}-R_{h} \mathbf{u}_{\tau}\right)+j\left(\mathbf{u}_{\varepsilon h \tau}\right)-2 j\left(\mathbf{u}_{\tau}\right) .
\end{aligned}
$$

Substituting the above inequality into (61), it yields that

$$
\begin{aligned}
\mu \| \mathbf{u}_{\varepsilon h}- & R_{h} \mathbf{u}\left\|_{V}^{2}+\varepsilon\right\| p_{\varepsilon h}-Q_{h} p \|^{2} \\
= & \underbrace{a\left(\mathbf{u}-R_{h} \mathbf{u}_{\varepsilon h}-\mathbf{u}_{h} \mathbf{u}\right)}_{I_{1}} \\
& +\underbrace{b\left(\mathbf{u}_{\varepsilon H}^{k}, \mathbf{u}_{\varepsilon H}^{k}, R_{h} \mathbf{u}-\mathbf{u}_{\varepsilon h}\right)-b\left(\mathbf{u}, \mathbf{u}, R_{h} \mathbf{u}-\mathbf{u}_{\varepsilon h}\right)}_{I_{2}} \\
& \underbrace{d\left(\mathbf{u}_{\varepsilon h}-R_{h} \mathbf{u}, p-Q_{h} p\right)+d\left(\mathbf{u}-R_{h} \mathbf{u}, p_{\varepsilon h}-Q_{h} p\right)}_{I_{3}} \\
& +\underbrace{\varepsilon\left(p_{\varepsilon H}^{k}-Q_{h} p, p_{\varepsilon h}-Q_{h} p\right)}_{I_{5}} \\
& +\underbrace{j\left(2 \mathbf{u}_{\tau}-R_{h} \mathbf{u}_{\tau}\right)-2 j\left(\mathbf{u}_{\tau}\right)+j\left(R_{h} \mathbf{u}_{\tau}\right) .}
\end{aligned}
$$

It follows from Hölder's inequality and Young's inequality that

$$
\begin{aligned}
I_{1}= & a\left(\mathbf{u}-R_{h} \mathbf{u}, \mathbf{u}_{\varepsilon h}-R_{h} \mathbf{u}\right) \leq \mu\left\|\mathbf{u}-R_{h} \mathbf{u}\right\|_{V}\left\|\mathbf{u}_{\varepsilon h}-R_{h} \mathbf{u}\right\|_{V} \\
\leq & \frac{\mu}{8}\left\|\mathbf{u}_{\varepsilon h}-R_{h} \mathbf{u}\right\|_{V}^{2}+2 \mu\left\|\mathbf{u}-R_{h} \mathbf{u}\right\|_{V}^{2}, \\
I_{3}= & d\left(\mathbf{u}-R_{h} \mathbf{u}, p_{\varepsilon h}-Q_{h} p\right)-d\left(\mathbf{u}_{\varepsilon h}-R_{h} \mathbf{u}, p-Q_{h} p\right) \\
\leq & \left\|\mathbf{u}-R_{h} \mathbf{u}\right\|_{V}\left\|p_{\varepsilon h}-Q_{h} p\right\|+\left\|\mathbf{u}_{\varepsilon h}-R_{h} \mathbf{u}\right\|_{V}\left\|p-Q_{h} p\right\| \\
\leq & \frac{\mu}{8}\left\|\mathbf{u}_{\varepsilon h}-R_{h} \mathbf{u}\right\|_{V}^{2}+\eta^{2}\left\|p_{\varepsilon h}-Q_{h} p\right\|^{2} \\
& +\frac{1}{\mu}\left\|p-Q_{h} p\right\|^{2}+\frac{1}{4 \eta^{2}}\left\|\mathbf{u}-R_{h} \mathbf{u}\right\|_{V}^{2},
\end{aligned}
$$

where $\eta>0$ is some small constant determined later. We rewrite $I_{2}$ as

$$
\begin{aligned}
I_{2}= & b\left(\mathbf{u}_{\varepsilon H}^{k}, \mathbf{u}_{\varepsilon H}^{k}, R_{h} \mathbf{u}-\mathbf{u}_{\varepsilon h}\right)-b\left(\mathbf{u}, \mathbf{u}, R_{h} \mathbf{u}-\mathbf{u}_{\varepsilon h}\right) \\
= & b\left(\mathbf{u}_{\varepsilon H}^{k}-\mathbf{u}, \mathbf{u}, R_{h} \mathbf{u}-\mathbf{u}_{\varepsilon h}\right)+b\left(\mathbf{u}, \mathbf{u}_{\varepsilon H}^{k}-\mathbf{u}, R_{h} \mathbf{u}-\mathbf{u}_{\varepsilon h}\right) \\
& +b\left(\mathbf{u}_{\varepsilon H}^{k}-\mathbf{u}, \mathbf{u}_{\varepsilon H}^{k}-u, R_{h} \mathbf{u}-\mathbf{u}_{\varepsilon h}\right) .
\end{aligned}
$$

Then using (13), (15), and Young's inequality, we can estimate $I_{2}$ by

$$
\begin{aligned}
I_{2} \leq & N\|\mathbf{u}\|_{2}\left\|R_{h} \mathbf{u}-\mathbf{u}_{\varepsilon h}\right\|_{V}\left\|\mathbf{u}_{\varepsilon H}^{k}-\mathbf{u}\right\| \\
& +N\left\|\mathbf{u}_{\varepsilon H}^{k}-\mathbf{u}\right\|_{V}^{2}\left\|R_{h} \mathbf{u}-\mathbf{u}_{\varepsilon h}\right\|_{V} \\
\leq & \frac{\mu}{8}\left\|R_{h} \mathbf{u}-\mathbf{u}_{\varepsilon h}\right\|_{V}^{2}+c\left(\left\|\mathbf{u}_{\varepsilon H}^{k}-\mathbf{u}\right\|^{2}+\left\|\mathbf{u}_{\varepsilon H}^{k}-\mathbf{u}\right\|_{V}^{4}\right) .
\end{aligned}
$$

It is easy to show that

$$
\begin{aligned}
I_{4} & =\varepsilon\left(p_{\varepsilon H}^{k}-Q_{h} p, p_{\varepsilon h}-Q_{h} p\right) \\
& \leq \varepsilon\left\|p_{\varepsilon H}^{k}-Q_{h} p\right\|\left\|p_{\varepsilon h}-Q_{h} p\right\| \\
& \leq \eta^{2}\left\|p_{\varepsilon h}-Q_{h} p\right\|^{2}+\frac{\varepsilon^{2}}{4 \eta^{2}}\left\|p_{\varepsilon H}^{k}-Q_{h} p\right\|^{2} \\
& \leq \eta^{2}\left\|p_{\varepsilon h}-Q_{h} p\right\|^{2}+\frac{\varepsilon^{2}}{2 \eta^{2}}\left(\left\|p-p_{\varepsilon H}^{k}\right\|^{2}+\left\|p-Q_{h} p\right\|^{2}\right) .
\end{aligned}
$$

Finally, from triangle inequality, $I_{5}$ is estimated by

$$
\begin{aligned}
I_{5}= & j\left(2 \mathbf{u}_{\tau}-R_{h} \mathbf{u}_{\tau}\right)-2 j\left(\mathbf{u}_{\tau}\right)+j\left(R_{h} \mathbf{u}_{\tau}\right) \\
& \leq 2 \int_{S} g\left|\mathbf{u}_{\tau}-R_{h} \mathbf{u}_{\tau}\right| d s \\
& \leq 2\|g\|_{L^{2}(S)}\left\|\mathbf{u}_{\tau}-R_{h} \mathbf{u}_{\tau}\right\|_{L^{2}(S)} .
\end{aligned}
$$

Substituting (65)-(69) into (64), it yields that

$$
\begin{aligned}
\left\|\mathbf{u}-\mathbf{u}_{\varepsilon h}\right\|_{V} & \\
\leq & \left\|\mathbf{u}-R_{h} \mathbf{u}\right\|_{V}+\left\|\mathbf{u}_{\varepsilon h}-R_{h} \mathbf{u}\right\|_{V} \\
\leq & \frac{2 \eta}{\sqrt{\mu}}\left\|p_{\varepsilon h}-Q_{h} p\right\| \\
& +c\left(\left\|\mathbf{u}-R_{h} \mathbf{u}\right\|_{V}+\left\|p-Q_{h} p\right\|+\left\|\mathbf{u}_{\tau}-R_{h} \mathbf{u}_{\tau}\right\|_{L^{2}(S)}^{1 / 2}\right. \\
& \left.\quad+\varepsilon\left\|p-p_{\varepsilon H}^{k}\right\|+\left\|\mathbf{u}-\mathbf{u}_{\varepsilon H}^{k}\right\|+\left\|\mathbf{u}-\mathbf{u}_{\varepsilon H}^{k}\right\|_{V}^{2}\right) \\
\leq & \frac{2 \eta}{\sqrt{\mu}}\left\|p_{\varepsilon h}-Q_{h} p\right\|+c\left(h^{5 / 4}+\varepsilon H^{5 / 4}+H^{9 / 4}+\varepsilon^{k+1}\right),
\end{aligned}
$$

where we use (24)-(26) and (57)-(58). Next, we estimate $\left\|p_{\varepsilon h}-Q_{h} p\right\|$. For all $\mathbf{w}_{h} \in V_{0 h}$, let $\mathbf{v}=\mathbf{u} \pm \mathbf{w}_{h}$ in (16) and $\mathbf{v}_{h}=\mathbf{u}_{\varepsilon h} \pm \mathbf{w}_{h}$ in (50), respectively. Then we get

$$
\begin{gathered}
a\left(\mathbf{u}, \mathbf{w}_{h}\right)+b\left(\mathbf{u}, \mathbf{u}, \mathbf{w}_{h}\right)-d\left(\mathbf{w}_{h}, p\right)=\left(\mathbf{f}, \mathbf{w}_{h}\right), \\
a\left(\mathbf{u}_{\varepsilon h}, \mathbf{w}_{h}\right)+b\left(\mathbf{u}_{\varepsilon H}^{k}, \mathbf{u}_{\varepsilon H}^{k}, \mathbf{w}_{h}\right)-d\left(\mathbf{w}_{h}, p_{\varepsilon h}\right)=\left(\mathbf{f}, \mathbf{w}_{h}\right) .
\end{gathered}
$$

Subtracting them and using (13), (15), we obtain

$$
\begin{aligned}
d\left(\mathbf{w}_{h}, p-p_{\varepsilon h}\right) & \\
= & a\left(\mathbf{u}-\mathbf{u}_{\varepsilon h}, \mathbf{w}_{h}\right)+b\left(\mathbf{u}, \mathbf{u}, \mathbf{w}_{h}\right)-b\left(\mathbf{u}_{\varepsilon H}^{k}, \mathbf{u}_{\varepsilon H}^{k}, \mathbf{w}_{h}\right) \\
= & a\left(\mathbf{u}-\mathbf{u}_{\varepsilon h}, \mathbf{w}_{h}\right)+b\left(\mathbf{u}, \mathbf{u}-\mathbf{u}_{\varepsilon H}^{k}, \mathbf{w}_{h}\right) \\
& +b\left(\mathbf{u}-\mathbf{u}_{\varepsilon H}^{k}, \mathbf{u}, \mathbf{w}_{h}\right)-b\left(\mathbf{u}-\mathbf{u}_{\varepsilon H}^{k}, \mathbf{u}-\mathbf{u}_{\varepsilon H}^{k}, \mathbf{w}_{h}\right) \\
\leq & \left(\mu\left\|\mathbf{u}-\mathbf{u}_{\varepsilon h}\right\|_{V}+N\|\mathbf{u}\|_{2}\left\|\mathbf{u}-\mathbf{u}_{\varepsilon H}^{k}\right\|\right. \\
& \left.+N\left\|\mathbf{u}-\mathbf{u}_{\varepsilon H}^{k}\right\|_{V}^{2}\right)\left\|\mathbf{w}_{h}\right\|_{V} .
\end{aligned}
$$


Therefore, it follows from (23) that $\left\|Q_{h} p-p_{\varepsilon h}\right\|$ can be estimated by

$$
\begin{aligned}
& \kappa\left\|Q_{h} p-p_{\varepsilon h}\right\| \\
& \leq \sup _{\mathbf{w}_{h} \in V_{0 h}} \frac{d\left(\mathbf{w}_{h}, Q_{h} p-p_{\varepsilon h}\right)}{\left\|\mathbf{w}_{h}\right\|_{V}} \\
&=\sup _{\mathbf{w}_{h} \in V_{0 h}} \frac{d\left(\mathbf{w}_{h}, Q_{h} p-p\right)+d\left(\mathbf{w}_{h}, p-p_{\varepsilon h}\right)}{\left\|\mathbf{w}_{h}\right\|_{V}} \\
& \leq\left\|p-Q_{h} p\right\|+\mu\left\|\mathbf{u}-\mathbf{u}_{\varepsilon h}\right\|_{V} \\
& \quad+N\|\mathbf{u}\|_{2}\left\|\mathbf{u}-\mathbf{u}_{\varepsilon H}^{k}\right\|+N\left\|\mathbf{u}-\mathbf{u}_{\varepsilon H}^{k}\right\|_{V}^{2} \\
& \leq \mu\left\|\mathbf{u}-\mathbf{u}_{\varepsilon h}\right\|_{V}+c\left(h^{5 / 4}+\varepsilon H^{5 / 4}+H^{9 / 4}+\varepsilon^{k+1}\right) .
\end{aligned}
$$

If we choose $\eta=\kappa / 4 \sqrt{\mu}$ such that $(2 \eta / \sqrt{\mu}) \cdot(\mu / \kappa)=1 / 2$, then substituting (73) into (70), we show

$$
\left\|\mathbf{u}-\mathbf{u}_{\varepsilon h}\right\|_{V} \leq c\left(h^{5 / 4}+\varepsilon H^{5 / 4}+H^{9 / 4}+\varepsilon^{k+1}\right) .
$$

From (73), again, we obtain

$$
\left\|p-p_{\varepsilon h}\right\| \leq c\left(h^{5 / 4}+\varepsilon H^{5 / 4}+H^{9 / 4}+\varepsilon^{k+1}\right) .
$$

Thus, we complete the proof of (59).

4.2. Two-Level Oseen Iteration Penalty Method. In Steps 1 and 2, we solve (48) and (49) on the coarse mesh, as in the following.

Step 1. Find $\left(\mathbf{u}_{\varepsilon H}^{0}, p_{\varepsilon H}^{0}\right) \in\left(V_{H}, M_{H}\right)$ by (48).

Step 2. For $k=1,2, \ldots$, find $\left(\mathbf{u}_{\varepsilon H}^{k}, p_{\varepsilon H}^{k}\right) \in\left(V_{H}, M_{H}\right)$ by (49).

In Step 3, we solve an Oseen type variational inequality problem on the fine mesh in terms of the Oseen iteration, as in the following.

Step 3. Find $\left(\mathbf{u}_{\varepsilon h}, p_{\varepsilon h}\right) \in\left(V_{h}, M_{h}\right)$ such that for all $\left(\mathbf{v}_{h}, q_{h}\right) \in$ $\left(V_{h}, M_{h}\right)$

$$
\begin{gathered}
a\left(\mathbf{u}_{\varepsilon h}, \mathbf{v}_{h}-\mathbf{u}_{\varepsilon h}\right)+b\left(\mathbf{u}_{\varepsilon H}^{k}, \mathbf{u}_{\varepsilon h}, \mathbf{v}_{h}-\mathbf{u}_{\varepsilon h}\right)+j\left(\mathbf{v}_{h \tau}\right) \\
-j\left(\mathbf{u}_{\varepsilon h \tau}\right)-d\left(\mathbf{v}_{h}-\mathbf{u}_{\varepsilon h}, p_{\varepsilon h}\right) \geq\left(\mathbf{f}, \mathbf{v}_{h}-\mathbf{u}_{\varepsilon h}\right), \\
d\left(\mathbf{u}_{\varepsilon h}, q_{h}\right)+\varepsilon\left(p_{\varepsilon h}, q_{h}\right)=\varepsilon\left(p_{\varepsilon H}^{k}, q_{h}\right) .
\end{gathered}
$$

From (12), it is easy to show that the solution $\left(\mathbf{u}_{\varepsilon h}, p_{\varepsilon h}\right)$ to the problem (76) satisfies

$$
\begin{aligned}
& \mu\left\|\mathbf{u}_{\varepsilon h}\right\|_{V}^{2}+\varepsilon\left\|p_{\varepsilon h}\right\|^{2} \\
& \leq\left(\mathbf{f}, \mathbf{u}_{\varepsilon h}\right)-j\left(\mathbf{u}_{\varepsilon h \tau}\right)+\varepsilon\left(p_{\varepsilon H}^{k}, p_{\varepsilon h}\right) \\
& \leq \kappa_{1}\left(\|\mathbf{f}\|+\|g\|_{L^{2}(S)}\right)\left\|\mathbf{u}_{\varepsilon h}\right\|_{V}+\varepsilon\left\|p_{\varepsilon H}^{k}\right\|\left\|p_{\varepsilon h}\right\| \\
& \leq \frac{\mu}{2}\left\|\mathbf{u}_{\varepsilon h}\right\|_{V}^{2}+\frac{\varepsilon}{2}\left\|p_{\varepsilon h}\right\|^{2}+\frac{\varepsilon}{2}\left\|p_{\varepsilon H}^{k}\right\|^{2} \\
&+\frac{\kappa_{1}^{2}}{2 \mu}\left(\|\mathbf{f}\|+\|g\|_{L^{2}(S)}\right)^{2} .
\end{aligned}
$$

Suppose that the initial data satisfies

$$
\frac{2 \sqrt{4 k+2} N \kappa_{1}}{\mu^{2}}\left(\|\mathbf{f}\|+\|g\|_{L^{2}(S)}\right)<1
$$

then using (52), we can estimate $\mathbf{u}_{\varepsilon h}$ by

$$
\begin{aligned}
\left\|\mathbf{u}_{\varepsilon h}\right\|_{V} & \leq \sqrt{\frac{\bar{\varepsilon}}{\mu}}\left\|p_{\varepsilon H}^{k}\right\|+\frac{\kappa_{1}}{\mu}\left(\|\mathbf{f}\|+\|g\|_{L^{2}(S)}\right) \\
& \leq\left(\sqrt{\frac{2 k+1}{2}}+1\right) \frac{\kappa_{1}}{\mu}\left(\|\mathbf{f}\|+\|g\|_{L^{2}(S)}\right) \\
& \leq \frac{\sqrt{4 k+2} \kappa_{1}}{\mu}\left(\|\mathbf{f}\|+\|g\|_{L^{2}(S)}\right) \leq \frac{\mu}{2 N} .
\end{aligned}
$$

For two-level Oseen iteration penalty method, the solution $\left(\mathbf{u}_{\varepsilon h}, p_{\varepsilon h}\right)$ is of the following error estimate.

Theorem 7. Suppose that the uniqueness condition (78) holds. Let $(\mathbf{u}, p) \in H^{3}(\Omega)^{2} \cap V \times H^{2}(\Omega) \cap M$ and $\left(\mathbf{u}_{\varepsilon h}, p_{\varepsilon h}\right) \in$ $\left(V_{h}, M_{h}\right)$ be the solutions to the problems (16) and (76), respectively; then they satisfy

$$
\left\|\mathbf{u}-\mathbf{u}_{\varepsilon h}\right\|_{V}+\left\|p-p_{\varepsilon h}\right\| \leq c\left(h^{5 / 4}+\varepsilon H^{5 / 4}+H^{9 / 4}+\varepsilon^{k+1}\right) .
$$

Proof. Proceeding as in the proof of (64), we can get

$$
\begin{aligned}
\mu \| \mathbf{u}_{\varepsilon h}- & R_{h} \mathbf{u}\left\|_{V}^{2}+\varepsilon\right\| p_{\varepsilon h}-Q_{h} p \|^{2} \\
= & \underbrace{a\left(\mathbf{u}-R_{h} \mathbf{u}, \mathbf{u}_{\varepsilon h}-R_{h} \mathbf{u}\right)}_{I_{1}} \\
& +\underbrace{b\left(\mathbf{u}_{\varepsilon H}^{k}, \mathbf{u}_{\varepsilon h}, R_{h} \mathbf{u}-\mathbf{u}_{\varepsilon h}\right)-b\left(\mathbf{u}, \mathbf{u}, R_{h} \mathbf{u}-\mathbf{u}_{\varepsilon h}\right)}_{I_{6}} \\
& \underbrace{d\left(\mathbf{u}_{\varepsilon h}-R_{h} \mathbf{u}, p-Q_{h} p\right)+d\left(\mathbf{u}-R_{h} \mathbf{u}, p_{\varepsilon h}-Q_{h} p\right)}_{I_{3}} \\
& +\underbrace{\varepsilon\left(p_{\varepsilon H}^{k}-Q_{h} p, p_{\varepsilon h}-Q_{h} p\right)}_{I_{4}} \\
& +\underbrace{j\left(2 \mathbf{u}_{\tau}-R_{h} \mathbf{u}_{\tau}\right)-2 j\left(\mathbf{u}_{\tau}\right)+j\left(R_{h} \mathbf{u}_{\tau}\right)}_{I_{5}} .
\end{aligned}
$$


In the above equation, $I_{1}, I_{3}, I_{4}$, and $I_{5}$ have been estimated in the proof of Theorem 6 . Here, we only estimate $I_{6}$. Using (12), (13), (15), and Young's inequality, we have

$$
\begin{aligned}
I_{6}= & b\left(\mathbf{u}_{\varepsilon H}^{k}, \mathbf{u}_{\varepsilon h}, R_{h} \mathbf{u}-\mathbf{u}_{\varepsilon h}\right)-b\left(\mathbf{u}, \mathbf{u}, R_{h} \mathbf{u}-\mathbf{u}_{\varepsilon h}\right) \\
= & b\left(\mathbf{u}_{\varepsilon H}^{k}, \mathbf{u}_{\varepsilon h}-R_{h} \mathbf{u}, R_{h} \mathbf{u}-\mathbf{u}_{\varepsilon h}\right) \\
& +b\left(\mathbf{u}_{\varepsilon H}^{k}, R_{h} \mathbf{u}-\mathbf{u}, R_{h} \mathbf{u}-\mathbf{u}_{\varepsilon h}\right) \\
& +b\left(\mathbf{u}_{\varepsilon H}^{k}-\mathbf{u}, \mathbf{u}, R_{h} \mathbf{u}-\mathbf{u}_{\varepsilon h}\right) \\
= & b\left(\mathbf{u}_{\varepsilon H}^{k}, R_{h} \mathbf{u}-\mathbf{u}, R_{h} \mathbf{u}-\mathbf{u}_{\varepsilon h}\right) \\
& +b\left(\mathbf{u}_{\varepsilon H}^{k}-\mathbf{u}, \mathbf{u}, R_{h} \mathbf{u}-\mathbf{u}_{\varepsilon h}\right) \\
\leq & N\left\|\mathbf{u}_{\varepsilon H}^{k}\right\|_{V}\left\|R_{h} \mathbf{u}-\mathbf{u}\right\|_{V}\left\|R_{h} \mathbf{u}-\mathbf{u}_{\varepsilon h}\right\|_{V} \\
& +N\|u\|_{2}\left\|R_{h} \mathbf{u}-\mathbf{u}_{\varepsilon h}\right\|_{V}\left\|\mathbf{u}_{\varepsilon H}^{k}-\mathbf{u}\right\| \\
\leq & \frac{\mu}{8}\left\|R_{h} \mathbf{u}-\mathbf{u}_{\varepsilon h}\right\|_{V}^{2}+c\left(\left\|\mathbf{u}_{\varepsilon H}^{k}-\mathbf{u}\right\|^{2}+\left\|R_{h} \mathbf{u}-\mathbf{u}\right\|_{V}^{2}\right) .
\end{aligned}
$$

Then substituting (65), (68), (69), and (82) into (81), it yields that

$$
\begin{aligned}
\left\|\mathbf{u}-\mathbf{u}_{\varepsilon h}\right\|_{V} \leq & \frac{2 \eta}{\sqrt{\mu}}\left\|p_{\varepsilon h}-Q_{h} p\right\| \\
& +c\left(h^{5 / 4}+\varepsilon H^{5 / 4}+H^{9 / 4}+\varepsilon^{k+1}\right) .
\end{aligned}
$$

In (83), we use (24)-(26) and (57)-(58). Next, we estimate $\left\|p_{\varepsilon h}-Q_{h} p\right\|$. For all $w_{h} \in V_{0 h}$, proceeding as in the proof of (72), from (51) and (78), we can show

$$
\begin{aligned}
d\left(\mathbf{w}_{h}, p-p_{\varepsilon h}\right) & =a\left(\mathbf{u}-\mathbf{u}_{\varepsilon h}, \mathbf{w}_{h}\right)+b\left(\mathbf{u}, \mathbf{u}, \mathbf{w}_{h}\right) \\
& -b\left(\mathbf{u}_{\varepsilon H}^{k}, \mathbf{u}_{\varepsilon h}, \mathbf{w}_{h}\right) \\
= & a\left(\mathbf{u}-\mathbf{u}_{\varepsilon h}, \mathbf{w}_{h}\right)+b\left(\mathbf{u}_{\varepsilon H}^{k}, \mathbf{u}-\mathbf{u}_{\varepsilon h}, \mathbf{w}_{h}\right) \\
& +b\left(\mathbf{u}-\mathbf{u}_{\varepsilon H}^{k}, \mathbf{u}, \mathbf{w}_{h}\right) \\
\leq & \left(\mu\left\|\mathbf{u}-\mathbf{u}_{\varepsilon h}\right\|_{V}+N\|\mathbf{u}\|_{2}\left\|\mathbf{u}-\mathbf{u}_{\varepsilon H}^{k}\right\|\right. \\
& \left.\quad+N\left\|\mathbf{u}_{\varepsilon H}^{k}\right\|_{V}\left\|\mathbf{u}-\mathbf{u}_{\varepsilon h}\right\|_{V}\right)\left\|\mathbf{w}_{h}\right\|_{V} \\
\leq & \left(\frac{5 \mu}{4}\left\|\mathbf{u}-\mathbf{u}_{\varepsilon h}\right\|_{V}+N\|\mathbf{u}\|_{2}\left\|\mathbf{u}-\mathbf{u}_{\varepsilon H}^{k}\right\|\right)\left\|\mathbf{w}_{h}\right\|_{V} .
\end{aligned}
$$

It follows from (23) and (84) that

$$
\begin{aligned}
\kappa\left\|Q_{h} p-p_{\varepsilon h}\right\| \\
\quad \leq \sup _{\mathbf{w}_{h} \in V_{0 h}} \frac{d\left(\mathbf{w}_{h}, Q_{h} p-p_{\varepsilon h}\right)}{\left\|\mathbf{w}_{h}\right\|_{V}} \\
\quad=\sup _{\mathbf{w}_{h} \in V_{0 h}} \frac{d\left(\mathbf{w}_{h}, Q_{h} p-p\right)+d\left(\mathbf{w}_{h}, p-p_{\varepsilon h}\right)}{\left\|\mathbf{w}_{h}\right\|_{V}} \\
\leq\left\|p-Q_{h} p\right\|+\frac{5 \mu}{4}\left\|\mathbf{u}-\mathbf{u}_{\varepsilon h}\right\|_{V}+N\|\mathbf{u}\|_{2}\left\|\mathbf{u}-\mathbf{u}_{\varepsilon H}^{k}\right\| \\
\leq \frac{5 \mu}{4}\left\|\mathbf{u}-\mathbf{u}_{\varepsilon h}\right\|_{V}+c\left(h^{5 / 4}+\varepsilon H^{5 / 4}+H^{9 / 4}+\varepsilon^{k+1}\right) .
\end{aligned}
$$

If we choose $\eta=\kappa / 5 \sqrt{\mu}$ such that $(2 \eta / \sqrt{\mu}) \cdot(5 \mu / 4 \kappa)=1 / 2$, then substituting (85) into (83), we show

$$
\left\|\mathbf{u}-\mathbf{u}_{\varepsilon h}\right\|_{V} \leq c\left(h^{5 / 4}+\varepsilon H^{5 / 4}+H^{9 / 4}+\varepsilon^{k+1}\right) .
$$

From (85), again, we obtain

$$
\left\|p-p_{\varepsilon h}\right\| \leq c\left(h^{5 / 4}+\varepsilon H^{5 / 4}+H^{9 / 4}+\varepsilon^{k+1}\right) .
$$

Thus, we complete the proof of (80).

4.3. Two-Level Newton Iteration Penalty Method. In Steps 1 and 2, we solve (48) and (49) on the coarse mesh, as in the following.

Step 1. Find $\left(\mathbf{u}_{\varepsilon H}^{0}, p_{\varepsilon H}^{0}\right) \in\left(V_{H}, M_{H}\right)$ by (48).

Step 2. For $k=1,2, \ldots$, find $\left(\mathbf{u}_{\varepsilon H}^{k}, p_{\varepsilon H}^{k}\right) \in\left(V_{H}, M_{H}\right)$ by (49).

In Step 3, we solve a linearized Navier-Stokes type variational inequality problem on the fine mesh in terms of the Newton iteration, as in the following.

Step 3. Find $\left(\mathbf{u}_{\varepsilon h}, p_{\varepsilon h}\right) \in\left(V_{h}, M_{h}\right)$ such that for all $\left(\mathbf{v}_{h}, q_{h}\right) \in$ $\left(V_{h}, M_{h}\right)$

$$
\begin{aligned}
& a\left(\mathbf{u}_{\varepsilon h}, \mathbf{v}_{h}-\mathbf{u}_{\varepsilon h}\right)+b\left(\mathbf{u}_{\varepsilon H}^{k}, \mathbf{u}_{\varepsilon h}, \mathbf{v}_{h}-\mathbf{u}_{\varepsilon h}\right) \\
&+b\left(\mathbf{u}_{\varepsilon h}, \mathbf{u}_{\varepsilon H}^{k}, \mathbf{v}_{h}-\mathbf{u}_{\varepsilon h}\right) \\
&+j\left(\mathbf{v}_{h \tau}\right)-j\left(\mathbf{u}_{\varepsilon h \tau}\right)-d\left(\mathbf{v}_{h}-\mathbf{u}_{\varepsilon h}, p_{\varepsilon h}\right) \\
& \geq\left(\mathbf{f}, \mathbf{v}_{h}-\mathbf{u}_{\varepsilon h}\right)+b\left(\mathbf{u}_{\varepsilon H}^{k}, \mathbf{u}_{\varepsilon H}^{k}, \mathbf{v}_{h}-\mathbf{u}_{\varepsilon h}\right), \\
& d\left(\mathbf{u}_{\varepsilon h}, q_{h}\right)+\varepsilon\left(p_{\varepsilon h}, q_{h}\right)=\varepsilon\left(p_{\varepsilon H}^{k}, q_{h}\right) .
\end{aligned}
$$

In this section, we will suppose that the initial data satisfies

$$
\frac{8 N \kappa_{1}}{\mu^{2}} \sqrt{\frac{2 k+1}{2}}\left(\|\mathbf{f}\|+\|g\|_{L^{2}(S)}\right)<1 .
$$

Then from (51), $\mathbf{u}_{\varepsilon H}^{k}$ satisfies $\left\|\mathbf{u}_{\varepsilon H}^{k}\right\|_{V} \leq \mu / 8 N$. Let $\mathbf{v}_{h}=$ $0, q_{h}=p_{\varepsilon h}$ in (88). Using (12), we obtain

$$
\begin{aligned}
& \mu\left\|\mathbf{u}_{\varepsilon h}\right\|_{V}^{2}-b\left(\mathbf{u}_{\varepsilon H}^{k}, \mathbf{u}_{\varepsilon h}, \mathbf{u}_{\varepsilon h}\right)+\varepsilon\left\|p_{\varepsilon h}\right\|^{2} \\
& \leq\left(\mathbf{f}, \mathbf{u}_{\varepsilon h}\right)-j\left(\mathbf{u}_{\varepsilon h \tau}\right)-b\left(\mathbf{u}_{\varepsilon H}^{k}, \mathbf{u}_{\varepsilon H}^{k}, \mathbf{u}_{\varepsilon h}\right)+\varepsilon\left(p_{\varepsilon H}^{k}, p_{\varepsilon h}\right) \\
& \leq \kappa_{1}\left(\|\mathbf{f}\|+\|g\|_{L^{2}(S)}\right)\left\|\mathbf{u}_{\varepsilon h}\right\|_{V} \\
&+N\left\|\mathbf{u}_{\varepsilon H}^{k}\right\|_{V}^{2}\left\|\mathbf{u}_{\varepsilon h}\right\|_{V}+\varepsilon\left\|p_{\varepsilon H}^{k}\right\|\left\|p_{\varepsilon h}\right\| \\
& \leq \frac{\mu}{2}\left\|\mathbf{u}_{\varepsilon h}\right\|_{V}^{2}+\frac{\varepsilon}{2}\left\|p_{\varepsilon h}\right\|^{2}+\frac{\varepsilon}{2}\left\|p_{\varepsilon H}^{k}\right\|^{2} \\
&+\frac{\kappa_{1}^{2}}{\mu}\left(\|\mathbf{f}\|+\|g\|_{L^{2}(S)}\right)^{2}+\frac{N^{2}}{\mu}\left\|\mathbf{u}_{\varepsilon H}^{k}\right\|_{V}^{4} .
\end{aligned}
$$

Since

$$
\begin{aligned}
& \mu\left\|\mathbf{u}_{\varepsilon h}\right\|_{V}^{2}-b\left(\mathbf{u}_{\varepsilon H}^{k}, \mathbf{u}_{\varepsilon h}, \mathbf{u}_{\varepsilon h}\right) \\
& \quad \geq \mu\left\|\mathbf{u}_{\varepsilon h}\right\|_{V}^{2}-N\left\|\mathbf{u}_{\varepsilon H}^{k}\right\|_{V}\left\|\mathbf{u}_{\varepsilon h}\right\|_{V}^{2} \geq \frac{7 \mu}{8}\left\|\mathbf{u}_{\varepsilon h}\right\|_{V}^{2},
\end{aligned}
$$


then

$$
\begin{aligned}
\left\|\mathbf{u}_{\varepsilon h}\right\|_{V} & \leq \frac{2 \kappa_{1}}{\sqrt{3} \mu}\left(\|\mathbf{f}\|+\|g\|_{L^{2}(S)}\right)+\frac{2 \sqrt{\varepsilon}}{\sqrt{3 \mu}}\left\|p_{\varepsilon H}^{k}\right\|+\frac{2 N}{\sqrt{3} \mu}\left\|\mathbf{u}_{\varepsilon H}^{k}\right\|_{V}^{2} \\
& \leq \frac{\sqrt{2}}{4 \sqrt{3}} \frac{1}{\sqrt{2 k+1}} \frac{\mu}{N}+\frac{1}{4 \sqrt{3}}\left\|\mathbf{u}_{\varepsilon H}^{k}\right\|_{V}+\frac{\mu}{4 \sqrt{3} N} \\
& <\left(\frac{1}{4}+\frac{1}{55}+\frac{1}{6}\right) \frac{\mu}{N}=\frac{574 \mu}{1320 N}<\frac{\mu}{2 N} .
\end{aligned}
$$

Theorem 8. Suppose that the uniqueness condition (89) holds. Let $(\mathbf{u}, p) \in H^{3}(\Omega)^{2} \cap V \times H^{2}(\Omega) \cap M$ and $\left(\mathbf{u}_{\varepsilon h}, p_{\varepsilon h}\right) \epsilon$ $\left(V_{h}, M_{h}\right)$ be the solutions to the problems (16) and (88), respectively; then they satisfy

$$
\left\|\mathbf{u}-\mathbf{u}_{\varepsilon h}\right\|_{V}+\left\|p-p_{\varepsilon h}\right\| \leq c\left(h^{5 / 4}+\varepsilon H^{5 / 4}+H^{5 / 2}+\varepsilon^{k+2}\right) .
$$

Proof. Proceeding as the in proof of (64), we can get

$$
\begin{aligned}
\mu \| \mathbf{u}_{\varepsilon h} & -R_{h} \mathbf{u}\left\|_{V}^{2}+\varepsilon\right\| p_{\varepsilon h}-Q_{h} p \|^{2} \\
= & \underbrace{a\left(\mathbf{u}-R_{h} \mathbf{u}, \mathbf{u}_{\varepsilon h}-R_{h} \mathbf{u}\right)}_{I_{1}} \\
& +\underbrace{-d\left(\mathbf{u}_{\varepsilon h}-R_{h} \mathbf{u}, p-Q_{h} p\right)+d\left(\mathbf{u}-R_{h} \mathbf{u}, p_{\varepsilon h}-Q_{h} p\right)}_{I_{3}} \\
& +\underbrace{\varepsilon\left(p_{\varepsilon H}^{k}-Q_{h} p, p_{\varepsilon h}-Q_{h} p\right)}_{I_{4}} \\
& +\underbrace{j\left(2 \mathbf{u}_{\tau}-R_{h} \mathbf{u}_{\tau}\right)-2 j\left(\mathbf{u}_{\tau}\right)+j\left(R_{h} \mathbf{u}_{\tau}\right)}_{I_{5}} \\
& +\underbrace{b\left(\mathbf{u}_{\varepsilon H}^{k}, \mathbf{u}_{\varepsilon h}, R_{h} \mathbf{u}-\mathbf{u}_{\varepsilon h}\right)+b\left(\mathbf{u}_{\varepsilon h}, \mathbf{u}_{\varepsilon H}^{k}, R_{h} \mathbf{u}-\mathbf{u}_{\varepsilon h}\right)-b\left(\mathbf{u}, \mathbf{u}, R_{h} \mathbf{u}-\mathbf{u}_{\varepsilon h}\right)-b\left(\mathbf{u}_{\varepsilon H}^{k}, \mathbf{u}_{\varepsilon H}^{k}, R_{h} \mathbf{u}-\mathbf{u}_{\varepsilon h}\right)}_{I_{7}} .
\end{aligned}
$$

In the above equation, $I_{1}, I_{3}, I_{4}$, and $I_{5}$ have been estimated in the proof of Theorem 6 . Here, we only estimate $I_{7}$. We rewrite $I_{7}$ as

$$
\begin{aligned}
I_{7}= & b\left(\mathbf{u}_{\varepsilon H}^{k}, \mathbf{u}_{\varepsilon h}, R_{h} \mathbf{u}-\mathbf{u}_{\varepsilon h}\right)+b\left(\mathbf{u}_{\varepsilon h}, \mathbf{u}_{\varepsilon H}^{k}, R_{h} \mathbf{u}-\mathbf{u}_{\varepsilon h}\right) \\
& -b\left(\mathbf{u}, \mathbf{u}, R_{h} \mathbf{u}-\mathbf{u}_{\varepsilon h}\right)-b\left(\mathbf{u}_{\varepsilon H}^{k}, \mathbf{u}_{\varepsilon H}^{k}, R_{h} \mathbf{u}-\mathbf{u}_{\varepsilon h}\right) \\
= & b\left(\mathbf{u}_{\varepsilon h}-\mathbf{u}, \mathbf{u}, R_{h} \mathbf{u}-\mathbf{u}_{\varepsilon h}\right)+b\left(\mathbf{u}_{\varepsilon h}, \mathbf{u}_{\varepsilon h}-\mathbf{u}, R_{h} \mathbf{u}-\mathbf{u}_{\varepsilon h}\right) \\
& -b\left(\mathbf{u}_{\varepsilon h}-\mathbf{u}_{\varepsilon H}^{k}, \mathbf{u}_{\varepsilon h}-\mathbf{u}_{\varepsilon H}^{k}, R_{h} \mathbf{u}-\mathbf{u}_{\varepsilon h}\right) \\
= & \underbrace{b\left(\mathbf{u}_{\varepsilon h}-R_{h} \mathbf{u}, \mathbf{u}, R_{h} \mathbf{u}-\mathbf{u}_{\varepsilon h}\right)+b\left(R_{h} \mathbf{u}-\mathbf{u}, \mathbf{u}, R_{h} \mathbf{u}-\mathbf{u}_{\varepsilon h}\right)}_{I_{g}} \\
& +\underbrace{b\left(\mathbf{u}_{\varepsilon h}, R_{h} \mathbf{u}-\mathbf{u}_{I_{h}} R_{h}-\mathbf{u}_{\varepsilon h}\right)}_{I_{10}} \\
& +\underbrace{b\left(R_{h} \mathbf{u}-\mathbf{u}_{\varepsilon h}, R_{h} \mathbf{u}-\mathbf{u}_{\varepsilon H}^{k}, R_{h} \mathbf{u}-\mathbf{u}_{\varepsilon h}\right)}_{I_{11}} \\
& +\underbrace{b\left(\mathbf{u}_{\varepsilon H}^{k}-R_{h} \mathbf{u}, R_{h} \mathbf{u}-\mathbf{u}_{\varepsilon H}^{k}, R_{h} \mathbf{u}-\mathbf{u}_{\varepsilon h}\right)} .
\end{aligned}
$$

$$
\begin{aligned}
& \leq \frac{\mu}{2}\left\|R_{h} \mathbf{u}-\mathbf{u}_{\varepsilon h}\right\|_{V}^{2}+\frac{\mu}{2}\left\|R_{h} \mathbf{u}-\mathbf{u}_{\varepsilon h}\right\|_{V}\left\|R_{h} \mathbf{u}-\mathbf{u}\right\|_{V} \\
& \leq \frac{\mu}{2}\left\|R_{h} \mathbf{u}-\mathbf{u}_{\varepsilon h}\right\|_{V}^{2}+\frac{\mu}{32}\left\|R_{h} \mathbf{u}-\mathbf{u}_{\varepsilon h}\right\|_{V}^{2}+4 \mu\left\|R_{h} \mathbf{u}-\mathbf{u}\right\|_{V}^{2} .
\end{aligned}
$$

Using (13) and (24), we estimate $I_{9}$ by

$$
\begin{aligned}
I_{9}= & b\left(\mathbf{u}_{\varepsilon h}, R_{h} \mathbf{u}-\mathbf{u}, R_{h} \mathbf{u}-\mathbf{u}_{\varepsilon h}\right) \\
= & b\left(\mathbf{u}_{\varepsilon h}-R_{h} \mathbf{u}, R_{h} \mathbf{u}-\mathbf{u}, R_{h} \mathbf{u}-\mathbf{u}_{\varepsilon h}\right) \\
& +b\left(R_{h} \mathbf{u}, R_{h} \mathbf{u}-\mathbf{u}, R_{h} \mathbf{u}-\mathbf{u}_{\varepsilon h}\right) \\
\leq & N\left\|R_{h} \mathbf{u}-\mathbf{u}\right\|_{V}\left\|R_{h} \mathbf{u}-\mathbf{u}_{\varepsilon h}\right\|_{V}^{2} \\
& +N\left\|R_{h} \mathbf{u}\right\|_{V}\left\|R_{h} \mathbf{u}-\mathbf{u}\right\|_{V}\left\|R_{h} \mathbf{u}-\mathbf{u}_{\varepsilon h}\right\|_{V} \\
\leq & C_{1} h^{2}\left\|R_{h} \mathbf{u}-\mathbf{u}_{\varepsilon h}\right\|_{V}^{2}+\frac{\mu}{32}\left\|R_{h} \mathbf{u}-\mathbf{u}_{\varepsilon h}\right\|_{V}^{2}+c\left\|R_{h} \mathbf{u}-\mathbf{u}\right\|_{V}^{2},
\end{aligned}
$$

From (13), (20), and Young's inequality, $I_{8}$ is estimated by

$$
\begin{aligned}
I_{8} & =b\left(\mathbf{u}_{\varepsilon h}-R_{h} \mathbf{u}, \mathbf{u}, R_{h} \mathbf{u}-\mathbf{u}_{\varepsilon h}\right)+b\left(R_{h} \mathbf{u}-\mathbf{u}, \mathbf{u}, R_{h} \mathbf{u}-\mathbf{u}_{\varepsilon h}\right) \\
& \leq N\|\mathbf{u}\|_{V}\left\|R_{h} \mathbf{u}-\mathbf{u}_{\varepsilon h}\right\|_{V}^{2}+N\|\mathbf{u}\|_{V}\left\|R_{h} \mathbf{u}-\mathbf{u}_{\varepsilon h}\right\|_{V}\left\|R_{h} \mathbf{u}-\mathbf{u}\right\|_{V}
\end{aligned}
$$

where $C_{1}>0$ is independent of $h, H$, and $\varepsilon$. Similarly, it follows from (13), (24), and (57) that

$$
\begin{aligned}
I_{10} & =b\left(R_{h} \mathbf{u}-\mathbf{u}_{\varepsilon h}, R_{h} \mathbf{u}-\mathbf{u}_{\varepsilon H}^{k}, R_{h} \mathbf{u}-\mathbf{u}_{\varepsilon h}\right) \\
& \leq N\left\|R_{h} \mathbf{u}-\mathbf{u}_{\varepsilon H}^{k}\right\|_{V}\left\|R_{h} \mathbf{u}-\mathbf{u}_{\varepsilon h}\right\|_{V}^{2}
\end{aligned}
$$




$$
\begin{aligned}
& \leq N\left(\left\|R_{h} \mathbf{u}-\mathbf{u}\right\|_{V}+\left\|\mathbf{u}-\mathbf{u}_{\varepsilon H}^{k}\right\|_{V}\right)\left\|R_{h} \mathbf{u}-\mathbf{u}_{\varepsilon h}\right\|_{V}^{2} \\
& \leq C_{2}\left(h^{2}+H^{5 / 4}+\varepsilon^{k+1}\right)\left\|R_{h} \mathbf{u}-\mathbf{u}_{\varepsilon h}\right\|_{V}^{2},
\end{aligned}
$$

where $C_{2}>0$ is independent of $h, H$, and $\varepsilon$. Finally, we can estimate $I_{11}$ by

$$
\begin{aligned}
I_{11} & =b\left(\mathbf{u}_{\varepsilon H}^{k}-R_{h} \mathbf{u}, R_{h} \mathbf{u}-\mathbf{u}_{\varepsilon H}^{k}, R_{h} \mathbf{u}-\mathbf{u}_{\varepsilon h}\right) \\
& \leq N\left\|R_{h} \mathbf{u}-\mathbf{u}_{\varepsilon H}^{k}\right\|_{V}^{2}\left\|R_{h} \mathbf{u}-\mathbf{u}_{\varepsilon h}\right\|_{V} \\
& \leq \frac{\mu}{32}\left\|R_{h} \mathbf{u}-\mathbf{u}_{\varepsilon h}\right\|_{V}^{2}+c\left(\left\|R_{h} \mathbf{u}-\mathbf{u}\right\|_{V}^{4}+\left\|\mathbf{u}-\mathbf{u}_{\varepsilon H}^{k}\right\|_{V}^{4}\right) .
\end{aligned}
$$

For sufficiently small $h, H$, and $\varepsilon$ such that $C_{1} h^{2}+C_{2}\left(h^{2}+\right.$ $\left.H^{5 / 4}+\varepsilon^{k+1}\right)=1 / 32$, substituting (65), (68), (69), and (95)(99) into (94), it yields that

$$
\begin{aligned}
\left\|\mathbf{u}-\mathbf{u}_{\varepsilon h}\right\|_{V} \leq & \frac{4 \eta}{\sqrt{\mu}}\left\|p_{\varepsilon h}-Q_{h} p\right\| \\
& +c\left(h^{5 / 4}+\varepsilon H^{5 / 4}+H^{5 / 2}+\varepsilon^{k+2}\right) .
\end{aligned}
$$

For all $w_{h} \in V_{0 h}$, proceeding as in the proof of (72), we can show

$$
\begin{aligned}
d\left(\mathbf{v}_{h}, p-p_{\varepsilon h}\right) & \\
= & a\left(\mathbf{u}-\mathbf{u}_{\varepsilon h}, \mathbf{v}_{h}\right)+b\left(\mathbf{u}, \mathbf{u}, \mathbf{v}_{h}\right)-b\left(\mathbf{u}_{\varepsilon H}^{k}, \mathbf{u}_{\varepsilon h}, \mathbf{v}_{h}\right) \\
& -b\left(\mathbf{u}_{\varepsilon h}, \mathbf{u}_{\varepsilon H}^{k}, \mathbf{v}_{h}\right)+b\left(\mathbf{u}_{\varepsilon H}^{k}, \mathbf{u}_{\varepsilon H}^{k}, \mathbf{v}_{h}\right) .
\end{aligned}
$$

Since

$$
\begin{aligned}
b\left(\mathbf{u}, \mathbf{u}, \mathbf{v}_{h}\right)- & b\left(\mathbf{u}_{\varepsilon H}^{k}, \mathbf{u}_{\varepsilon h}, \mathbf{v}_{h}\right)-b\left(u_{\varepsilon h}, \mathbf{u}_{\varepsilon H}^{k}, \mathbf{v}_{h}\right) \\
& +b\left(\mathbf{u}_{\varepsilon H}^{k}, \mathbf{u}_{\varepsilon H}^{k}, \mathbf{v}_{h}\right) \\
= & b\left(\mathbf{u}-\mathbf{u}_{\varepsilon h}, \mathbf{u}, \mathbf{v}_{h}\right)+b\left(\mathbf{u}, \mathbf{u}-R_{h} \mathbf{u}, v_{\mathbf{h}}\right) \\
& -b\left(\mathbf{u}-\mathbf{u}_{\varepsilon h}, \mathbf{u}-R_{h} \mathbf{u}, \mathbf{v}_{h}\right) \\
& +b\left(\mathbf{u}_{\varepsilon h}-\mathbf{u}_{\varepsilon H}^{k}, R_{h} \mathbf{u}-\mathbf{u}_{\varepsilon H}^{k}, \mathbf{v}_{h}\right) \\
& -b\left(\mathbf{u}_{\varepsilon H}^{k}, \mathbf{u}_{\varepsilon h}-R_{h} \mathbf{u}, \mathbf{v}_{h}\right) \\
\leq & \left(N\|\mathbf{u}\|_{V}\left\|\mathbf{u}-\mathbf{u}_{\varepsilon h}\right\|_{V}+N\|\mathbf{u}\|_{V}\left\|\mathbf{u}-R_{h} \mathbf{u}\right\|_{V}\right. \\
& \left.+\left\|\mathbf{u}-R_{h} \mathbf{u}\right\|_{V}\left\|\mathbf{u}-\mathbf{u}_{\varepsilon h}\right\|_{V}\right)\left\|\mathbf{v}_{h}\right\|_{V} \\
& +\left(\left\|\mathbf{u}-\mathbf{u}_{\varepsilon h}\right\|_{V}+\left\|\mathbf{u}-\mathbf{u}_{\varepsilon H}^{k}\right\|_{V}\right) \\
& \times\left(\left\|\mathbf{u}-R_{h} \mathbf{u}\right\|_{V}+\left\|\mathbf{u}-\mathbf{u}_{\varepsilon H}^{k}\right\|_{V}\right)\left\|\mathbf{v}_{h}\right\|_{V} \\
& +N\left\|\mathbf{u}_{\varepsilon H}^{k}\right\|_{V}\left(\left\|\mathbf{u}-\mathbf{u}_{\varepsilon h}\right\|_{V}+\left\|\mathbf{u}-R_{h} \mathbf{u}\right\|_{V}\right)\left\|\mathbf{v}_{h}\right\|_{V} \\
\leq & C_{3}\left(1+h^{2}+H^{2}+\varepsilon^{k+1}\right)\left\|\mathbf{u}-\mathbf{u}_{\varepsilon h}\right\|_{V}\left\|\mathbf{v}_{h}\right\|_{V} \\
+ & c\left(\left\|\mathbf{u}-R_{h} \mathbf{u}\right\|_{V}+\left\|\mathbf{u}-R_{h} \mathbf{u}\right\|_{V}^{2}+\left\|\mathbf{u}-\mathbf{u}_{\varepsilon H}^{k}\right\|_{V}^{2}\right)\left\|\mathbf{v}_{h}\right\|_{V} \\
& (102)
\end{aligned}
$$

where $C_{3}>0$ is independent of $h, H$, and $\varepsilon$, then from (23) we have

$$
\begin{gathered}
\kappa\left\|Q_{h} p-p_{\varepsilon h}\right\| \leq C_{3}\left(1+h^{2}+H^{2}+\varepsilon^{k+1}\right)\left\|\mathbf{u}-\mathbf{u}_{\varepsilon h}\right\|_{V} \\
+c\left(\left\|\mathbf{u}-R_{h} \mathbf{u}\right\|_{V}+\left\|p-Q_{h} p\right\|\right. \\
\left.+\left\|\mathbf{u}-R_{h} \mathbf{u}\right\|_{V}^{2}+\left\|\mathbf{u}-\mathbf{u}_{\varepsilon H}^{k}\right\|_{V}^{2}\right) .
\end{gathered}
$$

Thus, for sufficiently small $h, H, \varepsilon$, and $\eta$ such that

$$
\frac{C_{3}\left(1+h^{2}+H^{2}+\varepsilon^{k+1}\right)}{\kappa} \frac{4 \eta}{\sqrt{\mu}}<\frac{1}{2},
$$

substituting (103) into (100), we obtain

$$
\left\|\mathbf{u}-\mathbf{u}_{\varepsilon h}\right\|_{V} \leq c\left(h^{5 / 4}+\varepsilon H^{5 / 4}+H^{5 / 2}+\varepsilon^{k+2}\right) .
$$

From (103), again, we obtain

$$
\left\|p-p_{\varepsilon h}\right\| \leq c\left(h^{5 / 4}+\varepsilon H^{5 / 4}+H^{5 / 2}+\varepsilon^{k+2}\right) .
$$

Thus, we complete the proof of (93).

Remark 9. In terms of Theorems 6, 7, and 8, if we choose $\varepsilon=O(H), H=O\left(h^{5 / 9}\right)$ for the two-level Stokes or Oseen iteration penalty methods and $\varepsilon=O\left(H^{5 / 4}\right), H=O\left(h^{1 / 2}\right)$ for the two-level Newton iteration penalty method, then

$$
\left\|\mathbf{u}-\mathbf{u}_{\varepsilon h}\right\|_{V}+\left\|p-p_{\varepsilon h}\right\| \leq c h^{5 / 4} .
$$

4.4. An Improved Scheme. In this section, we will propose a scheme to improve the error estimates derived in Theorems $6-8$, which is described as follows.

In Steps 1 and 2, we solve (48) and (49) on the coarse mesh, as in the following.

Step 1. Find $\left(\mathbf{u}_{\varepsilon H}^{0}, p_{\varepsilon H}^{0}\right) \in\left(V_{H}, M_{H}\right)$ by (48).

Step 2. For $k=1,2, \ldots$, find $\left(\mathbf{u}_{\varepsilon H}^{k}, p_{\varepsilon H}^{k}\right) \in\left(V_{H}, M_{H}\right)$ by (49).

At Step 3, we solve a linearized problem (50) or (76) or (88) on the fine mesh in terms of Stokes iteration or Oseen iteration or Newton iteration, as in the following.

Step 3. Find $\left(\mathbf{u}_{\varepsilon h}, p_{\varepsilon h}\right) \in\left(V_{h}, M_{h}\right)$ by (50) or (76) or (88).

At Step 4, we solve a Newton correction of $\left(\mathbf{u}_{\varepsilon h}, p_{\varepsilon h}\right)$ on the fine mesh in terms of Newton iteration, as in the following.

Step 4. Find $\left(\mathbf{u}_{\varepsilon h}^{\star}, p_{\varepsilon h}^{\star}\right) \in\left(V_{h}, M_{h}\right)$ such that for all $\left(\mathbf{v}_{h}, q_{h}\right) \in$ $\left(V_{h}, M_{h}\right)$

$$
\begin{aligned}
a\left(\mathbf{u}_{\varepsilon h}^{\star}, \mathbf{v}_{h}-\mathbf{u}_{\varepsilon h}^{\star}\right)+b\left(\mathbf{u}_{\varepsilon h}, \mathbf{u}_{\varepsilon h}^{\star}, \mathbf{v}_{h}-\mathbf{u}_{\varepsilon h}^{\star}\right) \\
+b\left(\mathbf{u}_{\varepsilon h}^{\star}, \mathbf{u}_{\varepsilon h}, \mathbf{v}_{h}-\mathbf{u}_{\varepsilon h}^{\star}\right) \\
+j\left(\mathbf{v}_{h \tau}\right)-j\left(\mathbf{u}_{\varepsilon h \tau}^{\star}\right)-d\left(\mathbf{v}_{h}-\mathbf{u}_{\varepsilon h}^{\star}, p_{\varepsilon h}^{\star}\right) \\
\geq\left(\mathbf{f}, \mathbf{v}_{h}-\mathbf{u}_{\varepsilon h}^{\star}\right)+b\left(\mathbf{u}_{\varepsilon h}, \mathbf{u}_{\varepsilon h}, \mathbf{v}_{h}-\mathbf{u}_{\varepsilon h}^{\star}\right), \\
d\left(\mathbf{u}_{\varepsilon h}^{\star}, q_{h}\right)+\varepsilon\left(p_{\varepsilon h}^{\star}, q_{h}\right)=\varepsilon\left(p_{\varepsilon h}, q_{h}\right) .
\end{aligned}
$$

First, we show the following theorem. 
Theorem 10. Let $\left(\mathbf{u}_{\varepsilon h}^{k}, p_{\varepsilon h}^{k}\right)$ and $\left(\mathbf{u}_{\varepsilon h}^{\star}, p_{\varepsilon h}^{\star}\right)$ be the solutions of (28) and (108), respectively. Then there holds that

$$
\begin{aligned}
\| \mathbf{u}_{\varepsilon h}^{k} & -\mathbf{u}_{\varepsilon h}^{\star}\left\|_{V}+\right\| p_{\varepsilon h}^{k}-p_{\varepsilon h}^{\star} \| \\
& \leq c\left(\left\|\mathbf{u}_{\varepsilon h}^{k}-\mathbf{u}_{\varepsilon h}\right\|_{V}^{2}+\varepsilon^{1 / 2}\left\|p_{\varepsilon h}-p_{\varepsilon h}^{k-1}\right\|\right),
\end{aligned}
$$

where $\left(\mathbf{u}_{\varepsilon h}, p_{\varepsilon h}\right)$ is the solution to the problem (50) or (76) or (88).

Proof. Under the uniqueness condition (89), the solution $\mathbf{u}_{\varepsilon h}$ satisfies $\left\|\mathbf{u}_{\varepsilon h}\right\|_{V} \leq \mu / 2 N$. Taking $\mathbf{v}_{h}=\mathbf{u}_{\varepsilon h}^{\star}, q_{h}=p_{\varepsilon h}^{\star}-p_{\varepsilon h}^{k}$ in (28) and $\mathbf{v}_{h}=\mathbf{u}_{\varepsilon h}^{k}, q_{h}=p_{\varepsilon h}^{k}-p_{\varepsilon h}^{\star}$ in (108) and adding them yield

$$
\begin{aligned}
\mu \| \mathbf{u}_{\varepsilon h}^{\star}- & \mathbf{u}_{\varepsilon h}^{k}\left\|_{V}^{2}+\varepsilon\right\| p_{\varepsilon h}^{\star}-p_{\varepsilon h}^{k} \|^{2} \\
\leq & \varepsilon\left(p_{\varepsilon h}-p_{\varepsilon h}^{k-1}, p_{\varepsilon h}^{\star}-p_{\varepsilon h}^{k}\right)+b\left(\mathbf{u}_{\varepsilon h}^{k}, \mathbf{u}_{\varepsilon h}^{k}, \mathbf{u}_{\varepsilon h}^{\star}-\mathbf{u}_{\varepsilon h}^{k}\right) \\
& -b\left(\mathbf{u}_{\varepsilon h}, \mathbf{u}_{\varepsilon h}^{\star}, \mathbf{u}_{\varepsilon h}^{\star}-\mathbf{u}_{\varepsilon h}^{k}\right) \\
& -b\left(\mathbf{u}_{\varepsilon h}^{\star}, \mathbf{u}_{\varepsilon h}, \mathbf{u}_{\varepsilon h}^{\star}-\mathbf{u}_{\varepsilon h}^{k}\right)+b\left(\mathbf{u}_{\varepsilon h}, \mathbf{u}_{\varepsilon h}, \mathbf{u}_{\varepsilon h}^{\star}-\mathbf{u}_{\varepsilon h}^{k}\right) \\
= & \varepsilon\left(p_{\varepsilon h}-p_{\varepsilon h}^{k-1}, p_{\varepsilon h}^{\star}\right)+b\left(\mathbf{u}_{\varepsilon h}-\mathbf{u}_{\varepsilon h}^{k}, \mathbf{u}_{\varepsilon h}-\mathbf{u}_{\varepsilon h}^{k}, \mathbf{u}_{\varepsilon h}^{\star}-\mathbf{u}_{\varepsilon h}^{k}\right) \\
& +b\left(\mathbf{u}_{\varepsilon h}^{k}-\mathbf{u}_{\varepsilon h}^{\star}, \mathbf{u}_{\varepsilon h}, \mathbf{u}_{\varepsilon h}^{\star}-\mathbf{u}_{\varepsilon h}^{k}\right) .
\end{aligned}
$$

Using (13), Hölder's inequality, and Young's inequality, we obtain

$$
\begin{aligned}
\mu \| \mathbf{u}_{\varepsilon h}^{\star}- & \mathbf{u}_{\varepsilon h}^{k}\left\|_{V}^{2}+\varepsilon\right\| p_{\varepsilon h}^{\star}-p_{\varepsilon h}^{k} \|^{2} \\
\leq & \varepsilon\left\|p_{\varepsilon h}-p_{\varepsilon h}^{k-1}\right\|\left\|p_{\varepsilon h}^{\star}-p_{\varepsilon h}^{k}\right\|+N\left\|\mathbf{u}_{\varepsilon h}-\mathbf{u}_{\varepsilon h}^{k}\right\|_{V}^{2} \\
& \times\left\|\mathbf{u}_{\varepsilon h}^{\star}-\mathbf{u}_{\varepsilon h}^{k}\right\|_{V}+N\left\|\mathbf{u}_{\varepsilon h}\right\|\left\|_{V} \mathbf{u}_{\varepsilon h}^{\star}-\mathbf{u}_{\varepsilon h}^{k}\right\|_{V}^{2} \\
\leq & \frac{\varepsilon}{2}\left\|p_{\varepsilon h}^{\star}-p_{\varepsilon h}^{k}\right\|^{2}+\frac{\varepsilon}{2}\left\|p_{\varepsilon h}-p_{\varepsilon h}^{k-1}\right\|^{2}+\frac{\mu}{2}\left\|\mathbf{u}_{\varepsilon h}^{\star}-\mathbf{u}_{\varepsilon h}^{k}\right\|_{V}^{2} \\
& +\frac{\mu}{4}\left\|\mathbf{u}_{\varepsilon h}^{\star}-\mathbf{u}_{\varepsilon h}^{k}\right\|_{V}^{2}+\frac{N^{2}}{\mu}\left\|\mathbf{u}_{\varepsilon h}-\mathbf{u}_{\varepsilon h}^{k}\right\|_{V}^{4}
\end{aligned}
$$

That is,

$$
\left\|\mathbf{u}_{\varepsilon h}^{\star}-\mathbf{u}_{\varepsilon h}^{k}\right\|_{V} \leq \sqrt{\frac{2 \varepsilon}{\mu}}\left\|p_{\varepsilon h}-p_{\varepsilon h}^{k-1}\right\|+\frac{2 N}{\mu}\left\|\mathbf{u}_{\varepsilon h}-\mathbf{u}_{\varepsilon h}^{k}\right\|_{V}^{2} .
$$

For all $\mathbf{w}_{h} \in V_{0 h}$, taking $\mathbf{v}_{h}=\mathbf{u}_{\varepsilon h}^{k} \pm \mathbf{w}_{h}$ in the first inequality of (28) and $\mathbf{v}_{h}=\mathbf{u}_{\varepsilon h}^{\star} \pm \mathbf{w}_{h}$ in the first inequality of (108), it yields that

$$
\begin{gathered}
a\left(\mathbf{u}_{\varepsilon h}^{k}, \mathbf{w}_{h}\right)+b\left(\mathbf{u}_{\varepsilon h}^{k}, \mathbf{u}_{\varepsilon h}^{k}, \mathbf{w}_{h}\right)-d\left(\mathbf{w}_{h}, p_{\varepsilon h}^{k}\right)=\left(\mathbf{f}, \mathbf{w}_{h}\right), \\
a\left(\mathbf{u}_{\varepsilon h}^{\star}, \mathbf{w}_{h}\right)+b\left(\mathbf{u}_{\varepsilon h}, \mathbf{u}_{\varepsilon h}^{\star}, \mathbf{w}_{h}\right)+b\left(\mathbf{u}_{\varepsilon h}^{\star}, \mathbf{u}_{\varepsilon h}, \mathbf{w}_{h}\right) \\
-d\left(\mathbf{w}_{h}, p_{\varepsilon h}^{\star}\right)=\left(\mathbf{f}, \mathbf{w}_{h}\right)+b\left(\mathbf{u}_{\varepsilon h}, \mathbf{u}_{\varepsilon h}, \mathbf{w}_{h}\right) .
\end{gathered}
$$

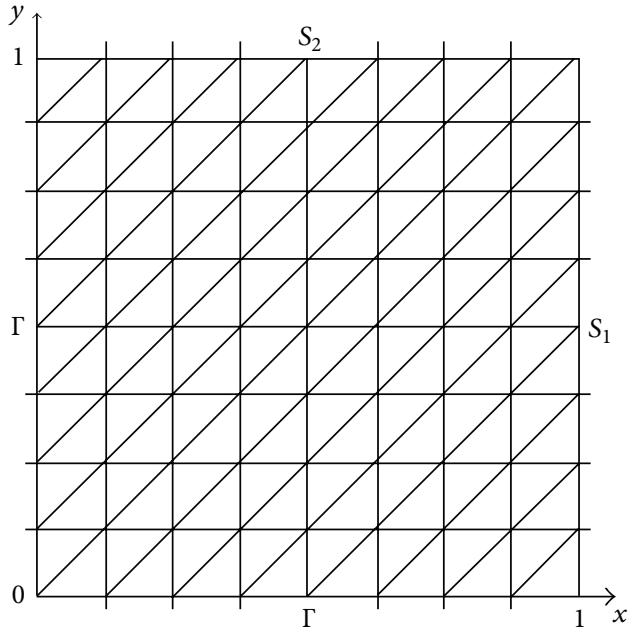

FIgURE 1: Domain $\Omega$.

Subtracting them and using (23), (112), we obtain

$$
\begin{aligned}
\kappa \| & p_{\varepsilon h}^{\star}-p_{\varepsilon h}^{k} \| \\
\leq & \sup _{\mathbf{w}_{h} \in V_{0 h}} \frac{d\left(\mathbf{w}_{h}, p_{\varepsilon h}^{\star}-p_{\varepsilon h}^{k}\right)}{\left\|\mathbf{w}_{h}\right\|_{V}} \\
= & \sup _{\mathbf{w}_{h} \in V_{0 h}} \frac{a\left(\mathbf{u}_{\varepsilon h}^{\star}-\mathbf{u}_{\varepsilon h}^{k}, \mathbf{w}_{h}\right)+b\left(\mathbf{u}_{\varepsilon h}, \mathbf{u}_{\varepsilon h}^{\star}, \mathbf{w}_{h}\right)}{\left\|\mathbf{w}_{h}\right\|_{V}} \\
& +\sup _{\mathbf{w}_{h} \in V_{0 h}} \frac{b\left(\mathbf{u}_{\varepsilon h}^{\star}, \mathbf{u}_{\varepsilon h}, \mathbf{w}_{h}\right)-b\left(\mathbf{u}_{\varepsilon h}^{k}, \mathbf{u}_{\varepsilon h}^{k}, \mathbf{w}_{h}\right)-b\left(\mathbf{u}_{\varepsilon h}, \mathbf{u}_{\varepsilon h}, \mathbf{w}_{h}\right)}{\left\|\mathbf{w}_{h}\right\|_{V}} \\
= & \sup _{\mathbf{w}_{h} \in V_{0 h}} \frac{a\left(\mathbf{u}_{\varepsilon h}^{\star}-\mathbf{u}_{\varepsilon h}^{k}, \mathbf{w}_{h}\right)+b\left(\mathbf{u}_{\varepsilon h}^{\star}-\mathbf{u}_{\varepsilon h}^{k}, \mathbf{u}_{\varepsilon h}, \mathbf{w}_{h}\right)}{\left\|\mathbf{w}_{h}\right\|_{V}} \\
& +\sup _{\mathbf{w}_{h} \in V_{0 h}} \frac{b\left(\mathbf{u}_{\varepsilon h}, \mathbf{u}_{\varepsilon h}^{\star}-\mathbf{u}_{\varepsilon h}^{k}, \mathbf{w}_{h}\right)-b\left(\mathbf{u}_{\varepsilon h}^{k}-\mathbf{u}_{\varepsilon h}, \mathbf{u}_{\varepsilon h}^{k}-\mathbf{u}_{\varepsilon h}, \mathbf{w}_{h}\right)}{\left\|\mathbf{w}_{h}\right\|_{V}} \\
\leq & \mu\left\|\mathbf{u}_{\varepsilon h}^{\star}-\mathbf{u}_{\varepsilon h}^{k}\right\|_{V}+2 N\left\|\mathbf{u}_{\varepsilon h}\right\|_{V}\left\|\mathbf{u}_{\varepsilon h}^{\star}-\mathbf{u}_{\varepsilon h}^{k}\right\|_{V} \\
\leq & 2 \sqrt{2 \mu \varepsilon}\left\|p_{\varepsilon h}-p_{\varepsilon h}^{k-1}\right\|+5 N\left\|\mathbf{u}_{\varepsilon h}^{k}-\mathbf{u}_{\varepsilon h}\right\|_{V}^{2} . \\
& +N\left\|\mathbf{u}_{\varepsilon h}^{k}-\mathbf{u}_{\varepsilon h}\right\|_{V}^{2} \\
\leq & 2 \mu \mathbf{u}_{\varepsilon h}^{\star}-\mathbf{u}_{\varepsilon h}^{k}\|\|_{V}+N\left\|\mathbf{u}_{\varepsilon h}^{k}-\mathbf{u}_{\varepsilon h}\right\|_{V}^{2} \\
&
\end{aligned}
$$

From (34) and Theorems 6-10, we get the following error estimates.

Theorem 11. Let $(\mathbf{u}, p) \in H^{3}(\Omega)^{2} \cap V \times H^{2}(\Omega) \cap M$ and $\left(\mathbf{u}_{\varepsilon h}^{\star}, p_{\varepsilon h}^{\star}\right) \in\left(V_{h}, M_{h}\right)$ be the solutions to the problems (16) 

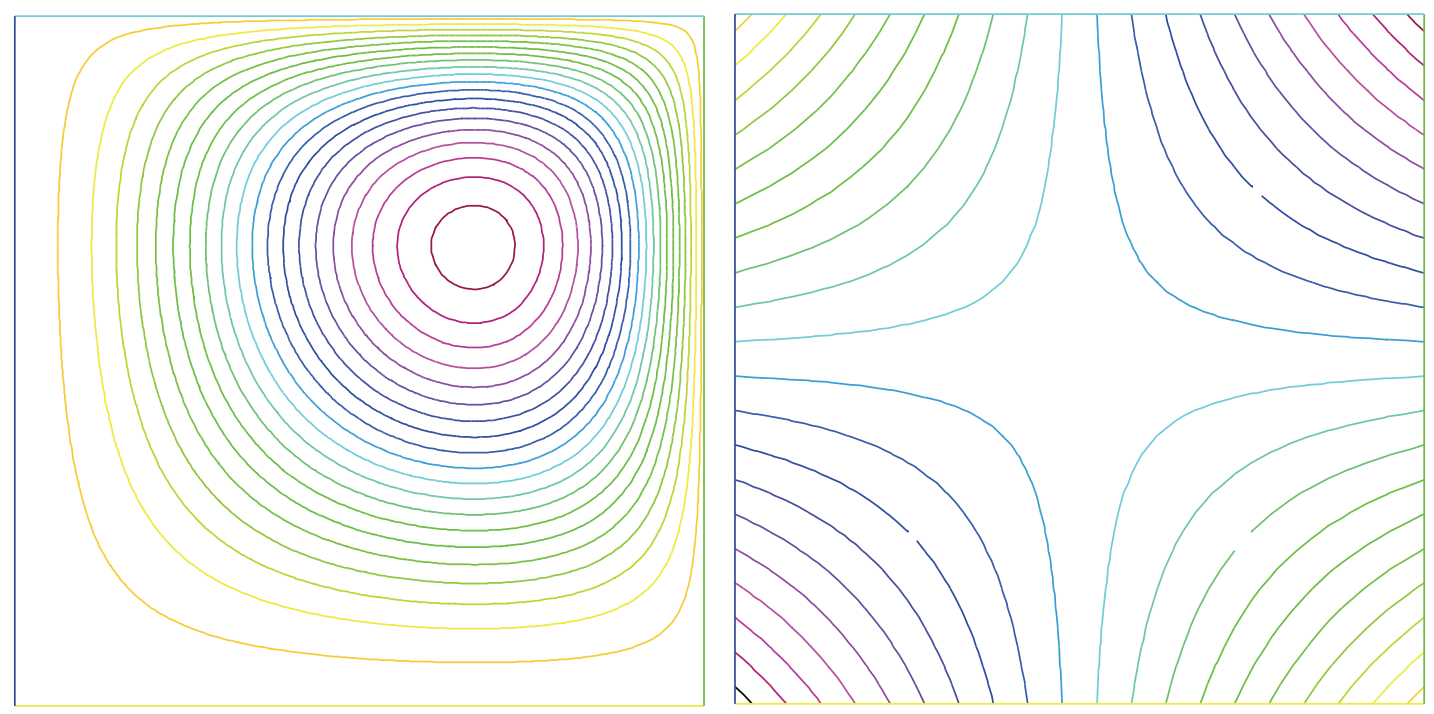

\begin{tabular}{|c|c|}
\hline IsoValue & $\begin{array}{l}\text { IsoValue } \\
0.0115226\end{array}$ \\
\hline 0.00164605 & - 0.01262 \\
\hline - 0.00274345 & - 0.0137174 \\
\hline . 0.00384085 & - 0.0148148 \\
\hline - 0.00493824 & - 0.0159122 \\
\hline - 0.00603564 & - 0.0170096 \\
\hline - 0.00713304 & - 0.018107 \\
\hline - 0.00823044 & - 0.0192044 \\
\hline - 0.00932783 & - 0.0203018 \\
\hline - 0.0104252 & - 0.021399 \\
\hline
\end{tabular}

\begin{tabular}{|c|c|}
\hline IsoValue & IsoValue \\
\hline - -0.95 & \\
\hline$=-0.85$ & - 0.15 \\
\hline$=-0.75$ & - 0.25 \\
\hline - -0.65 & - 0.35 \\
\hline - -0.55 & - 0.45 \\
\hline - -0.45 & - 0.55 \\
\hline . -0.35 & - 0.65 \\
\hline - -0.25 & - 0.75 \\
\hline - -0.15 & - 0.85 \\
\hline . -0.05 & - 0.95 \\
\hline
\end{tabular}

FIGURE 2: Streamline of flow and pressure contour for exact solution.

and (108), respectively. Then for the two-level Stokes or Oseen iteration penalty methods, they satisfy

$$
\begin{aligned}
\| \mathbf{u}- & \mathbf{u}_{\varepsilon h}^{\star}\left\|_{V}+\right\| p-p_{\varepsilon h}^{\star} \| \\
& \leq c\left(h^{5 / 4}+H^{9 / 2}+\varepsilon^{3 / 2} H^{5 / 4}+\varepsilon^{1 / 2} H^{9 / 4}+\varepsilon^{k+1 / 2}\right) .
\end{aligned}
$$

And for the two-level Newton iteration penalty method, they satisfy

$$
\begin{aligned}
& \left\|\mathbf{u}-\mathbf{u}_{\varepsilon h}^{\star}\right\|_{V}+\left\|p-p_{\varepsilon h}^{\star}\right\| \\
& \quad \leq c\left(h^{5 / 4}+H^{5}+\varepsilon^{3 / 2} H^{5 / 4}+\varepsilon^{1 / 2} H^{5 / 2}+\varepsilon^{k+1 / 2}\right) .
\end{aligned}
$$

Remark 12. If we choose $H=O\left(h^{5 / 18}\right), \varepsilon=O\left(h^{5 / 4}\right)$ in (115) for two-level Stokes or Oseen iteration penalty methods and $H=O\left(h^{1 / 4}\right), \varepsilon=O\left(h^{5 / 4}\right)$ in (116) for the two-level Newton iteration penalty method, then we obtain

$$
\left\|\mathbf{u}-\mathbf{u}_{\varepsilon h}^{\star}\right\|_{V}+\left\|p-p_{\varepsilon h}^{\star}\right\| \leq c h^{5 / 4} .
$$

\section{Numerical Results}

In this section, we will give numerical results to confirm the error analysis obtained in Section 4. Since these two-level Stokes/Oseen/Newton iteration penalty methods are given in the form of the variational inequality problems which are not directly solved, the appropriate iteration algorithm must be constructed. Here we use the Uzawa iteration algorithm introduced in [28].

For simplicity, we only give the Uzawa iteration method for solving the variational inequality problem (16). Similar schemes can be used to solve the twolevel Stokes/Oseen/Newton iteration penalty schemes in Section 4. First, there exists a multiplier $\lambda \in \Lambda$ such that the variational inequality problem (16) is equivalent to the following variational identity problem:

$$
\begin{gathered}
a(\mathbf{u}, \mathbf{v})+b(\mathbf{u}, \mathbf{u}, \mathbf{v})-d(\mathbf{v}, p)+\int_{S} \lambda g \mathbf{v}_{\tau} d s=(\mathbf{f}, \mathbf{v}), \\
\forall \mathbf{v} \in V, \\
d(\mathbf{u}, q)=0, \quad \forall q \in M, \\
\lambda u_{\tau}=\left|u_{\tau}\right|, \quad \text { a.e. on } S,
\end{gathered}
$$

where $\lambda \in \Lambda=\left\{\gamma \in L^{2}(S):|\gamma(x)| \leq 1\right.$ a.e. on $\left.S\right\}$. In this case, we can solve the problem (16) by the following Uzawa iteration scheme:

$$
\lambda^{0} \in \Lambda \text { is given; }
$$



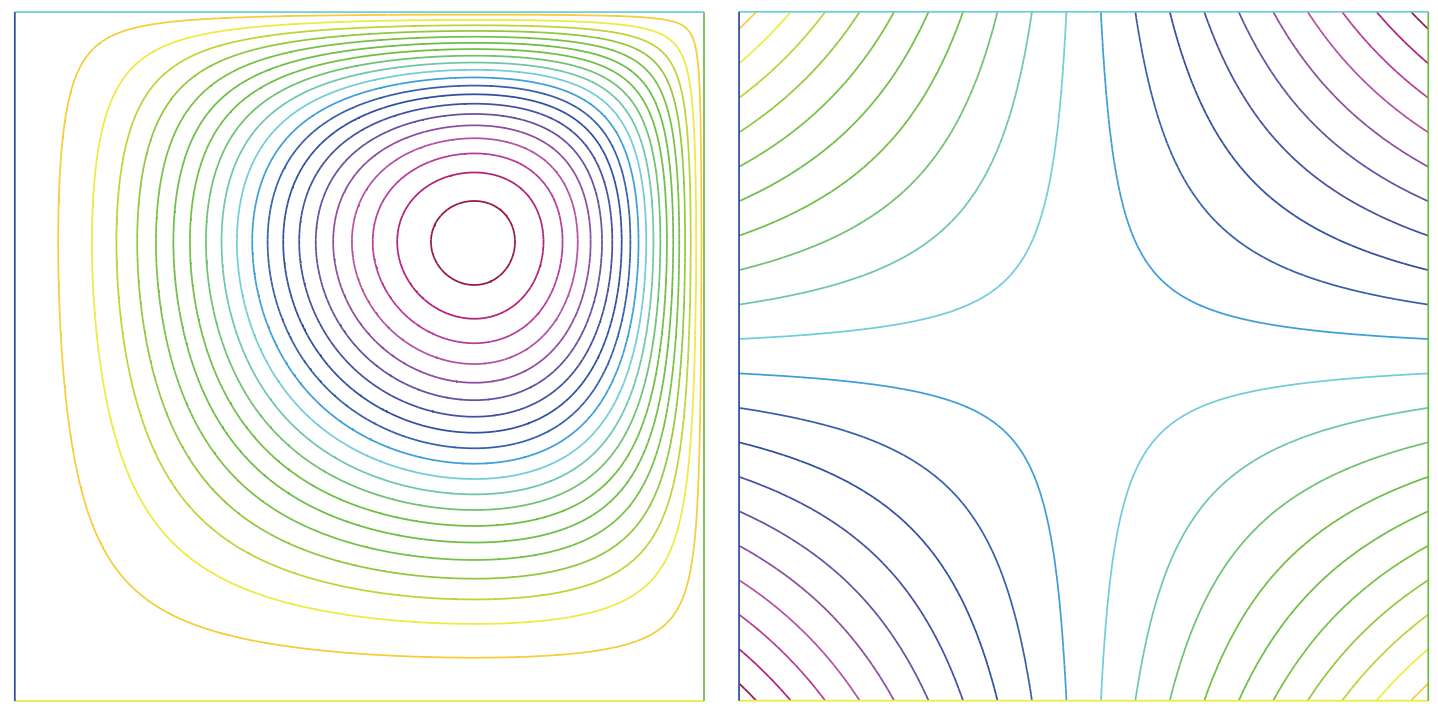

\begin{tabular}{|c|c|}
\hline IsoValue & $\begin{array}{l}\text { IsoValue } \\
0.0115226\end{array}$ \\
\hline - 0.000548696 & - 0.0115226 \\
\hline$=0.00164609$ & - 0.01262 \\
\hline - 0.00274348 & - 0.0137174 \\
\hline - 0.00384087 & - 0.0148148 \\
\hline - 0.00493827 & - 0.0159122 \\
\hline - 0.00603566 & - 0.0170096 \\
\hline - 0.00713305 & - 0.018107 \\
\hline - 0.00823044 & - 0.0192044 \\
\hline . 0.00932784 & - 0.0203018 \\
\hline - 0.0104252 & - 0.0213992 \\
\hline
\end{tabular}

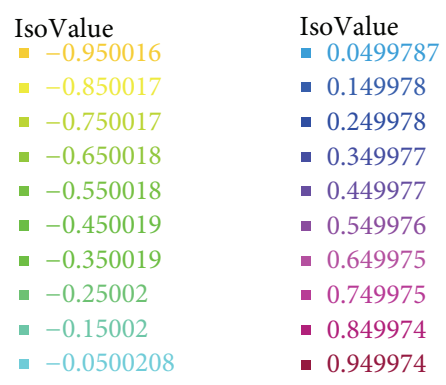

FIGURE 3: Streamline of flow and pressure contour by Stokes method.

then $\lambda^{n}$ is known; we compute $\left(\mathbf{u}^{n}, p^{n}\right)$ and $\lambda^{n+1}$ by

$$
\begin{gathered}
a\left(\mathbf{u}^{n}, \mathbf{v}\right)+b\left(\mathbf{u}^{n}, \mathbf{u}^{n}, \mathbf{v}\right)-d\left(\mathbf{v}, p^{n}\right) \\
=(\mathbf{f}, \mathbf{v})-\int_{S} \lambda^{n} g \mathbf{v}_{\tau} d s, \quad \forall \mathbf{v} \in V, \\
d\left(\mathbf{u}^{n}, q\right)=0, \quad \forall q \in M, \\
\lambda^{n+1}=P_{\Lambda}\left(\lambda^{n}+\rho g \mathbf{u}_{\tau}^{n}\right), \quad \rho>0,
\end{gathered}
$$

where

$$
P_{\Lambda}(\gamma)=\sup (-1, \inf (1, \gamma)), \quad \forall \gamma \in L^{2}(S) .
$$

Consider the problems (1)-(2) in the fixed square domain $(0,1) \times(0,1)$ (see Figure 1$)$. Let $\mu=0.1$. The external force $f$ is chosen such that the exact solution $(\mathbf{u}, p)$ is

$$
\begin{gathered}
\mathbf{u}(x, y)=\left(u_{1}(x, y), u_{2}(x, y)\right), \\
p(x, y)=(2 x-1)(2 y-1), \\
u_{1}(x, y)=-x^{2} y(x-1)(3 y-2), \\
u_{2}(x, y)=x y^{2}(y-1)(3 x-2) .
\end{gathered}
$$

It is easy to verify that the exact solution $\mathbf{u}$ satisfies $\mathbf{u}=0$ on $\Gamma, \mathbf{u} \cdot \mathbf{n}=u_{1}=0, u_{2} \neq 0$ on $S_{1}$ and $u_{1} \neq 0, \mathbf{u} \cdot \mathbf{n}=u_{2}=0$ on
$S_{2}$. Moreover, the tangential vector $\tau$ on $S_{1}$ and $S_{2}$ are $(0,1)$ and $(-1,0)$. Thus, we have

$$
\begin{array}{ll}
\sigma_{\tau}=4 \mu y^{2}(y-1) & \text { on } S_{1}, \\
\sigma_{\tau}=4 \mu x^{2}(x-1) & \text { on } S_{2} .
\end{array}
$$

On the other hand, from the nonlinear slip boundary conditions (2), there holds that

$$
\left|\sigma_{\tau}\right| \leq g
$$

then the function $g$ can be chosen as $g=-\sigma_{\tau} \geq 0$ on $S_{1}$ and $S_{2}$.

In all experiments, we choose $\mu=0.1$, iteration initial value $\lambda^{0}=1$, and $\rho=\mu / 2$. In terms of Theorems 6 and 7 , for the two-level Stokes/Oseen penalty iteration methods, there holds that

$$
\left\|\mathbf{u}-\mathbf{u}_{\varepsilon h}\right\|_{V}+\left\|p-p_{\varepsilon h}\right\| \leq c\left(h^{5 / 4}+\varepsilon H^{5 / 4}+H^{9 / 4}+\varepsilon^{k+1}\right) .
$$

Then we choose $\varepsilon=O(H), H=O\left(h^{5 / 9}\right), k=2$ such that

$$
\left\|\mathbf{u}-\mathbf{u}_{\varepsilon h}\right\|_{V}+\left\|p-p_{\varepsilon h}\right\| \leq c h^{5 / 4} \text {. }
$$

We pick eight coarse mesh size values; that is, $H=$ $1 / 4,1 / 6,1 / 8, \ldots, 1 / 18$. In Table 1 , the scaling between $1 / H$ and $1 / h=(1 / H)^{9 / 5}$ is given. 

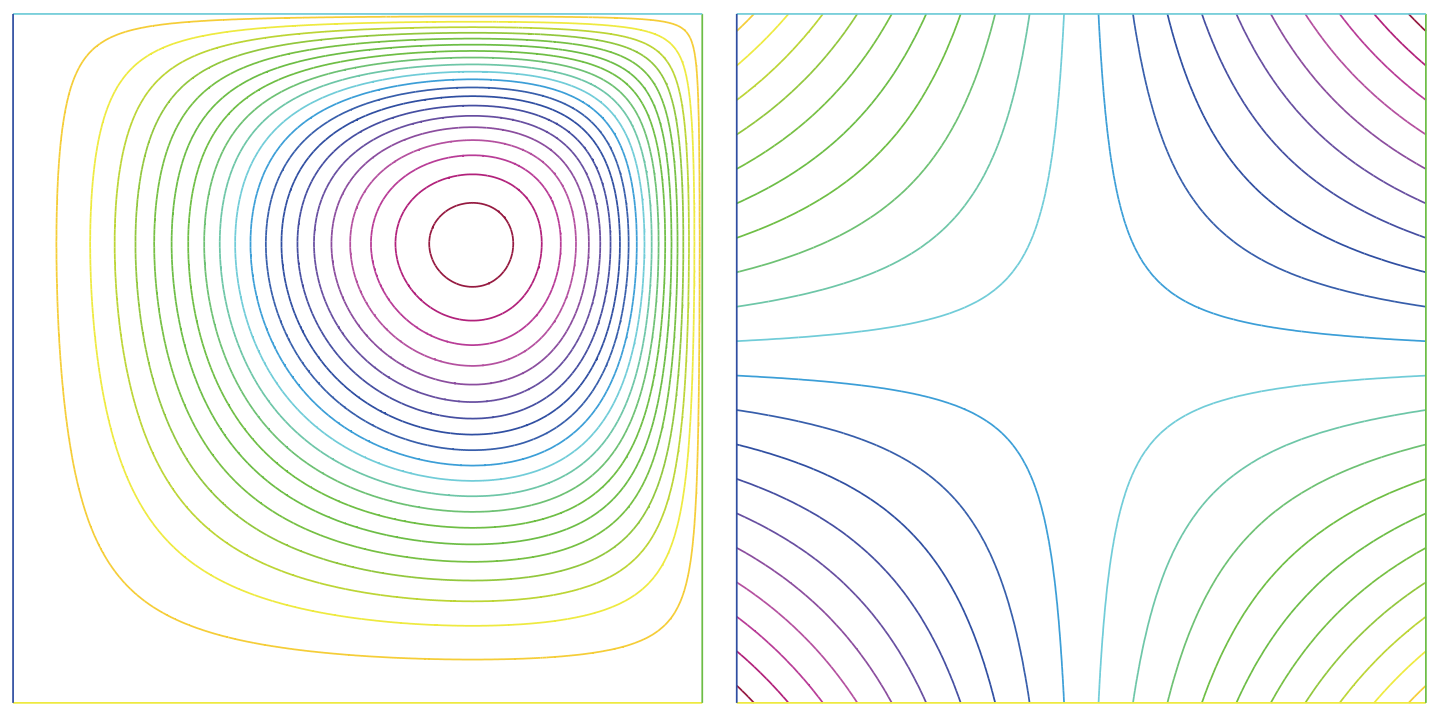

\begin{tabular}{|c|c|}
\hline $\begin{array}{l}\text { IsoValue } \\
0.000548696\end{array}$ & $\begin{array}{l}\text { IsoValue } \\
0.0115226\end{array}$ \\
\hline 0.00164609 & - 0.01262 \\
\hline 0.00274348 & - 0.0137174 \\
\hline . 0.00384088 & - 0.0148148 \\
\hline - 0.00493827 & - 0.0159122 \\
\hline . 0.00603566 & - 0.0170096 \\
\hline . 0.00713305 & - 0.018107 \\
\hline - 0.00823045 & - 0.0192044 \\
\hline . 0.00932784 & - 0.0203018 \\
\hline . 0.0104252 & - 0.021399 \\
\hline
\end{tabular}

\begin{tabular}{|c|c|}
\hline $\begin{array}{l}\text { IsoValue } \\
-0.950015\end{array}$ & $\begin{array}{l}\text { IsoValue } \\
0.0499791\end{array}$ \\
\hline$=-0.850016$ & - 0.149979 \\
\hline = -0.750017 & - 0.249978 \\
\hline - -0.650017 & - 0.349977 \\
\hline - -0.550018 & - 0.449977 \\
\hline . -0.450018 & - 0.549976 \\
\hline - -0.350019 & - 0.649976 \\
\hline - -0.250019 & - 0.749975 \\
\hline - -0.15002 & - 0.849975 \\
\hline - -0.0500204 & - 0.949974 \\
\hline
\end{tabular}

FiguRE 4: Streamline of flow and pressure contour by Oseen method.
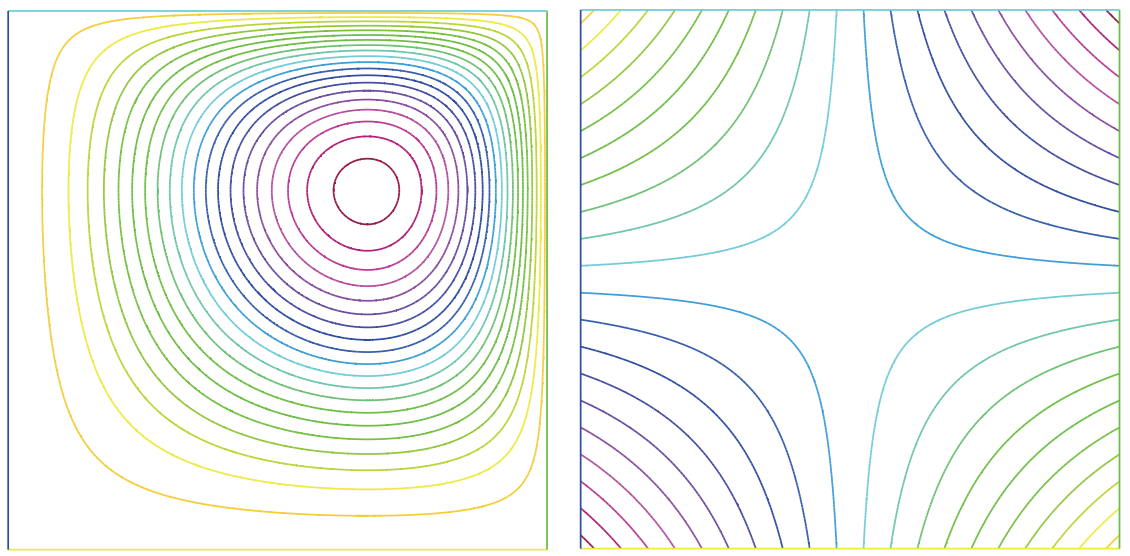

\begin{tabular}{|c|c|c|c|}
\hline $\begin{array}{l}\text { IsoValue } \\
0.000548697\end{array}$ & $\begin{array}{l}\text { IsoValue } \\
0.0115226\end{array}$ & $\begin{array}{l}\text { IsoValue } \\
\square-0.949997\end{array}$ & $\begin{array}{l}\text { IsoValue } \\
0.0499936\end{array}$ \\
\hline 0.00164609 & - 0.01262 & = -0.849998 & - 0.149993 \\
\hline 0.00274349 & - 0.0137174 & - -0.749999 & - 0.249992 \\
\hline - 0.00384088 & - 0.0148148 & . -0.65 & - 0.349991 \\
\hline - 0.00493828 & - 0.0159122 & - -0.550001 & - 0.44999 \\
\hline - 0.00603567 & - 0.0170096 & . -0.450002 & - 0.549989 \\
\hline - 0.00713307 & - 0.018107 & - -0.350003 & - 0.649988 \\
\hline . 0.00823046 & - 0.0192044 & - -0.250004 & - 0.749987 \\
\hline . 0.00932785 & - 0.0203018 & . -0.150005 & - 0.849986 \\
\hline 0.0104252 & - 0.0213992 & . -0.0500055 & - 0.949986 \\
\hline
\end{tabular}

Figure 5: Streamline of flow and pressure contour by Newton method. 
TABLE 1: Comparison of the scaling between $1 / H$ and $1 / h$.

\begin{tabular}{lcccccccc}
\hline $1 / H$ & 4 & 6 & 8 & 10 & 12 & 14 & 16 & 18 \\
\hline $1 / h$ & 12.125 & 25.157 & 42.224 & 63.095 & 87.604 & 115.619 & 147.033 & 181.756 \\
\hline
\end{tabular}

TABLE 2: Numerical relative error for velocity with $H=1 / 14$ and $h=1 / 115$.

\begin{tabular}{lcccc}
\hline$\varepsilon_{0}$ & 0.01 & 0.001 & 0.0001 & 0.00001 \\
\hline Stokes & $1.04374 e-03$ & $1.84283 e-04$ & $1.52977 e-04$ & $1.52631 e-04$ \\
Oseen & $1.04345 e-03$ & $1.78281 e-04$ & $1.45638 e-04$ & $1.45275 e-04$ \\
\hline
\end{tabular}

TABle 3: Numerical relative error for pressure with $H=1 / 14$ and $h=1 / 115$.

\begin{tabular}{lcccc}
\hline$\varepsilon_{0}$ & 0.01 & 0.001 & 0.0001 & 0.00001 \\
\hline Stokes & $1.85222 e-04$ & $6.20827 e-05$ & $5.96578 e-05$ & $5.96412 e-05$ \\
Oseen & $1.85112 e-04$ & $6.13519 e-05$ & $5.88604 e-05$ & $5.88398 e-05$ \\
\hline
\end{tabular}

TABLE 4: Numerical relative error for Stokes method.

\begin{tabular}{lccccc}
\hline $1 / H$ & $1 / h$ & $\left\|\mathbf{u}-\mathbf{u}_{\varepsilon h}\right\|_{V} /\|\mathbf{u}\|_{V}$ & $\left\|p-p_{\varepsilon h}\right\| /\|p\|$ & Iteration & $\mathrm{CPU}(\mathrm{s})$ \\
\hline 4 & 12 & $1.51517 e-02$ & $5.41171 e-03$ & 2 & 0.281 \\
6 & 25 & $3.30825 e-03$ & $1.25062 e-03$ & 2 & 0.889 \\
8 & 42 & $1.15862 e-03$ & $4.44992 e-04$ & 2 & 2.302 \\
10 & 63 & $5.25908 e-04$ & $1.99251 e-04$ & 2 & 5.038 \\
12 & 87 & $2.91391 e-04$ & $1.05862 e-04$ & 2 & 9.806 \\
14 & 115 & $1.84283 e-04$ & $6.20827 e-05$ & 2 & 17.621 \\
16 & 147 & $1.30602 e-04$ & $3.96161 e-05$ & 2 & 40.549 \\
18 & 181 & $1.01944 e-04$ & $2.77123 e-05$ & 2 & 53.445 \\
\hline \multicolumn{7}{l}{ Order } & 1.727 & 1.913 & \\
\hline \multicolumn{7}{l}{}
\end{tabular}

Setting $\varepsilon=\varepsilon_{0} H$, the comparison of relative error $\left\|\mathbf{u}-\mathbf{u}_{\varepsilon h}\right\|_{V} /\|\mathbf{u}\|_{V}$ and $\left\|p-p_{\varepsilon h}\right\| /\|p\|$ for different $\varepsilon_{0}>$ 0 is shown in Tables 2 and 3 when we use the two-level Stokes/Oseen penalty iteration methods with $1 / H=14$ and $1 / h=115$. We can see that, for our present testing case, it suffices to set $\varepsilon=0.001 H$ if it is hoped to be as large as possible.

Thus, set $\varepsilon=0.001 H$ and $1 / h \approx(1 / H)^{9 / 5}$. Tables 4 and 5 display the relative $H^{1}$ errors of the velocity and the relative $L^{2}$ errors of the pressure and their average convergence orders and CPU time when we use the twolevel Stokes iteration penalty method and two-level Oseen iteration penalty method, respectively. Based on Tables 4 and 5 , the two-level Stokes/Oseen iteration penalty methods can reach the convergence orders of $O\left(h^{5 / 4}\right)$ for both velocity and pressure, in $H^{1}$ - and $L^{2}$-norms, respectively, as shown in (126).

Next, we give the numerical results by using the two-level Newton iteration penalty method. In terms of Theorem 8 , there holds that

$$
\left\|\mathbf{u}-\mathbf{u}_{\varepsilon h}\right\|_{V}+\left\|p-p_{\varepsilon h}\right\| \leq c\left(h^{5 / 4}+\varepsilon H^{5 / 4}+H^{5 / 2}+\varepsilon^{k+2}\right) .
$$

TABLE 5: Numerical relative error for Oseen method.

\begin{tabular}{lccccc}
\hline $1 / H$ & $1 / h$ & $\left\|\mathbf{u}-\mathbf{u}_{\varepsilon h}\right\|_{V} /\|\mathbf{u}\|_{V}$ & $\left\|p-p_{\varepsilon h}\right\| /\|p\|$ & Iteration & $\mathrm{CPU}(\mathrm{s})$ \\
\hline 4 & 12 & $1.49411 e-02$ & $5.38449 e-03$ & 2 & 0.316 \\
6 & 25 & $3.23052 e-03$ & $1.24245 e-03$ & 2 & 1.006 \\
8 & 42 & $1.12567 e-03$ & $4.41332 e-04$ & 2 & 2.613 \\
10 & 63 & $5.08693 e-04$ & $1.93710 e-04$ & 2 & 5.728 \\
12 & 87 & $2.81455 e-04$ & $1.04715 e-04$ & 2 & 11.213 \\
14 & 115 & $1.78281 e-04$ & $6.13519 e-05$ & 2 & 20.045 \\
16 & 147 & $1.26872 e-04$ & $3.91207 e-05$ & 2 & 42.242 \\
18 & 181 & $9.95916 e-05$ & $2.73676 e-05$ & 2 & 59.391 \\
\hline \multicolumn{7}{l}{ Order } & 1.728 & 1.915 &
\end{tabular}

TABLE 6: Numerical relative error for Newton method.

\begin{tabular}{lccccc}
\hline $1 / H$ & $1 / h$ & $\left\|\mathbf{u}-\mathbf{u}_{\varepsilon h}\right\|_{V} /\|\mathbf{u}\|_{V}$ & $\left\|p-p_{\varepsilon h}\right\| /\|p\|$ & Iteration & $\mathrm{CPU}(\mathrm{s})$ \\
\hline 4 & 16 & $8.10332 e-03$ & $3.02474 e-03$ & 2 & 0.535 \\
6 & 36 & $1.51165 e-03$ & $5.98186 e-04$ & 2 & 2.256 \\
8 & 64 & $4.76536 e-04$ & $1.89983 e-04$ & 2 & 6.991 \\
10 & 100 & $2.08297 e-04$ & $8.02333 e-05$ & 2 & 16.977 \\
12 & 144 & $1.10901 e-04$ & $3.89471 e-05$ & 2 & 37.804 \\
14 & 196 & $7.20875 e-05$ & $2.21184 e-05$ & 2 & 78.811 \\
\hline \multicolumn{7}{l}{ Order } & 1.811 & 1.947 &
\end{tabular}

Then we choose $\varepsilon=0.01 H^{5 / 4}$ and $1 / h=(1 / H)^{2}$ such that

$$
\left\|\mathbf{u}-\mathbf{u}_{\varepsilon h}\right\|_{V}+\left\|p-p_{\varepsilon h}\right\| \leq c h^{5 / 4} \text {. }
$$

Because when $H=1 / 16$ and $h=1 / 256$, this method does not work and the computer displays "out of memory". Thus, in this experiment, we pick six coarse mesh size values; that is, $H=1 / 4,1 / 6, \ldots, 1 / 14$. Table 6 displays the relative $H^{1}$ errors of the velocity and the relative $L^{2}$ errors of the pressure and their average convergence orders and CPU time when we use the two-level Newton iteration penalty method. Based on Tables 4 and 5, we can see that the two-level Newton iteration penalty method also reaches the convergence orders of $\mathrm{O}\left(h^{5 / 4}\right)$ for both velocity and pressure, in $H^{1}$ - and $L^{2}$ norms, respectively, as shown in (128).

Figures 2, 3, 4, and 5 show the streamline of flow and the pressure contour of the numerical solution by the twolevel Stokes/Oseen/Newton iteration penalty methods and the exact solution, respectively.

\section{Acknowledgments}

This work is supported by the National Natural Science Foundation of China under Grant nos. 10901122, 11001205 and by Zhejiang Provincial Natural Science Foundation of China under Grant no. LY12A01015.

\section{References}

[1] H. Fujita, "Flow Problems with Unilateral Boundary conditions," Leçcons, Collèege de France, 1993.

[2] H. Fujita, "A mathematical analysis of motions of viscous incompressible fluid under leak or slip boundary conditions," RIMS Kokyuroku, no. 888, pp. 199-216, 1994. 
[3] H. Fujita, "Non-stationary Stokes flows under leak boundary conditions of friction type," Journal of Computational Mathematics, vol. 19, no. 1, pp. 1-8, 2001.

[4] H. Fujita, "A coherent analysis of Stokes flows under boundary conditions of friction type," Journal of Computational and Applied Mathematics, vol. 149, no. 1, pp. 57-69, 2002.

[5] Y. Li and K. Li, "Existence of the solution to stationary NavierStokes equations with nonlinear slip boundary conditions," Journal of Mathematical Analysis and Applications, vol. 381, no. 1, pp. 1-9, 2011.

[6] Y. Li and K. Li, "Global strong solutions of two-dimensional Navier-Stokes equations with nonlinear slip boundary conditions," Journal of Mathematical Analysis and Applications, vol. 393, no. 1, pp. 1-13, 2012.

[7] N. Saito and H. Fujita, "Regularity of solutions to the Stokes equation under a certain nonlinear boundary condition, The Navier-Stokes Equations," in Proceedings of the 2nd International Conference on the Navier Stokes-Equations: Theory and Numerical Methods, vol. 223 of Dekker Lecture Notes in Pure and Applied Mathematics, pp. 73-86, 2001.

[8] N. Saito, "On the Stokes equation with the leak and slip boundary conditions of friction type: regularity of solutions," Publications of the Research Institute for Mathematical Sciences, Kyoto University, vol. 40, no. 2, pp. 345-383, 2004.

[9] Y. He and J. Li, "Convergence of three iterative methods based on the finite element discretization for the stationary NavierStokes equations," Computer Methods in Applied Mechanics and Engineering, vol. 198, no. 15-16, pp. 1351-1359, 2009.

[10] R. An and H. L. Qiu, "Two-Level Newton iteration methods for Navier-Stokes type variational inequality problem," The Advances in Applied Mathematics and Mechanics, vol. 5, no. 1, pp. 36-54, 2013.

[11] V. Girault and J.-L. Lions, "Two-grid finite-element schemes for the steady Navier-Stokes problem in polyhedra," Portugaliae Mathematica, vol. 58, no. 1, pp. 25-57, 2001.

[12] Y. He and K. Li, "Two-level stabilized finite element methods for the steady Navier-Stokes problem," Computing, vol. 74, no. 4, pp. 337-351, 2005.

[13] W. Layton and H. W. J. Lenferink, "A multilevel mesh independence principle for the Navier-Stokes equations," SIAM Journal on Numerical Analysis, vol. 33, no. 1, pp. 17-30, 1996.

[14] Y. Li and R. An, "Two-level pressure projection finite element methods for Navier-Stokes equations with nonlinear slip boundary conditions," Applied Numerical Mathematics, vol. 61, no. 3, pp. 285-297, 2011.

[15] K. Li and Y. Hou, "An AIM and one-step Newton method for the Navier-Stokes equations," Computer Methods in Applied Mechanics and Engineering, vol. 190, no. 46-47, pp. 6141-6155, 2001.

[16] M. Marion and J. Xu, "Error estimates on a new nonlinear Galerkin method based on two-grid finite elements," SIAM Journal on Numerical Analysis, vol. 32, no. 4, pp. 1170-1184, 1995.

[17] J. Xu, “Two-grid discretization techniques for linear and nonlinear PDEs," SIAM Journal on Numerical Analysis, vol. 33, no. 5, pp. 1759-1777, 1996.

[18] R. Temam, Navier-Stokes Equations: Theory and Numerical analysis, AMS Chelsea, Providence, RI, USA, 2001.

[19] Y. Li and R. An, "Penalty finite element method for NavierStokes equations with nonlinear slip boundary conditions," International Journal for Numerical Methods in Fluids, vol. 69, no. 3, pp. 550-566, 2012.
[20] X. X. Dai, P. P. Tang, and M. H. Wu, "Analysis of an iterative penalty method for Navier-Stokes equations with nonlinear slip boundary conditions," International Journal for Numerical Methods in Fluids, vol. 72, no. 4, pp. 403-413, 2013.

[21] X. L. Cheng and A. W. Shaikh, "Analysis of the iterative penalty method for the Stokes equations," Applied Mathematics Letters, vol. 19, no. 10, pp. 1024-1028, 2006.

[22] X. Dai, "Finite element approximation of the pure Neumann problem using the iterative penalty method," Applied Mathematics and Computation, vol. 186, no. 2, pp. 1367-1373, 2007.

[23] J. Shen, "On error estimates of the penalty method for unsteady Navier-Stokes equations," SIAM Journal on Numerical Analysis, vol. 32, no. 2, pp. 386-403, 1995.

[24] I. Babuška, "The finite element method with Lagrangian multipliers," Numerische Mathematik, vol. 20, pp. 179-192, 1972/73.

[25] F. Brezzi, "On the existence, uniqueness and approximation of saddle-point problems arising from Lagrangian multipliers," Revue Française d'Automatique, Informatique, Recherche Opérationnelle, vol. 8, no. 2, pp. 129-151, 1974.

[26] S. C. Brenner and L. R. Scott, The Mathematical Theory of Finite Element Methods, vol. 15 of Texts in Applied Mathematics, Springer, New York, NY, USA, 2nd edition, 2002.

[27] R. Glowinski, Numerical Methods for Nonlinear Variational Problems, Scientific Computation, Springer, Berlin, Germany, 2008.

[28] Y. Li and K. Li, "Uzawa iteration method for Stokes type variational inequality of the second kind," Acta Mathematicae Applicatae Sinica, vol. 27, no. 2, pp. 303-315, 2011. 


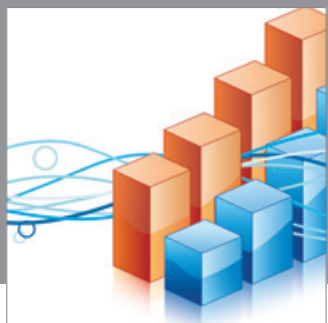

Advances in

Operations Research

mansans

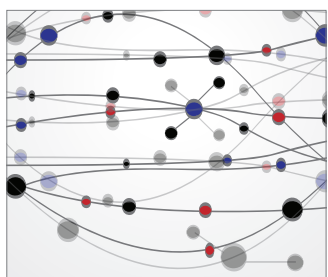

The Scientific World Journal
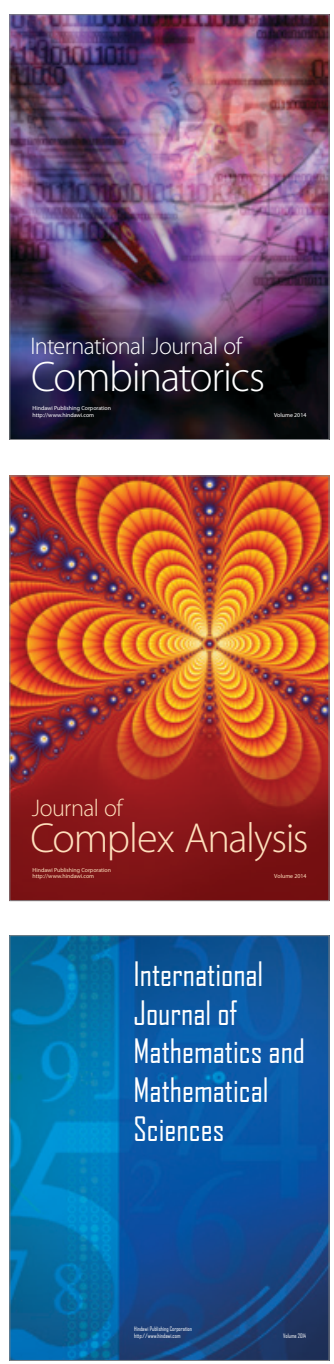
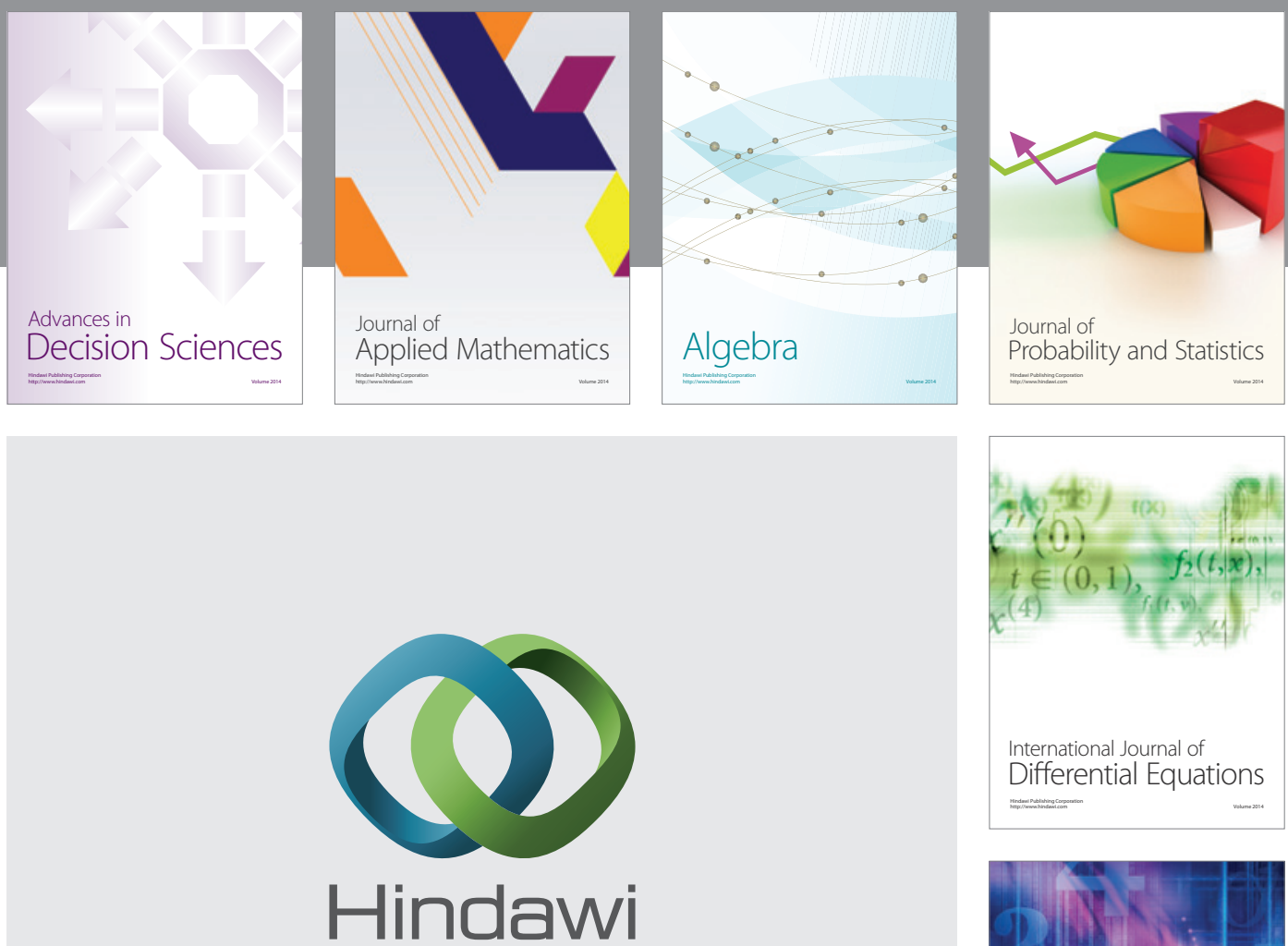

Submit your manuscripts at http://www.hindawi.com
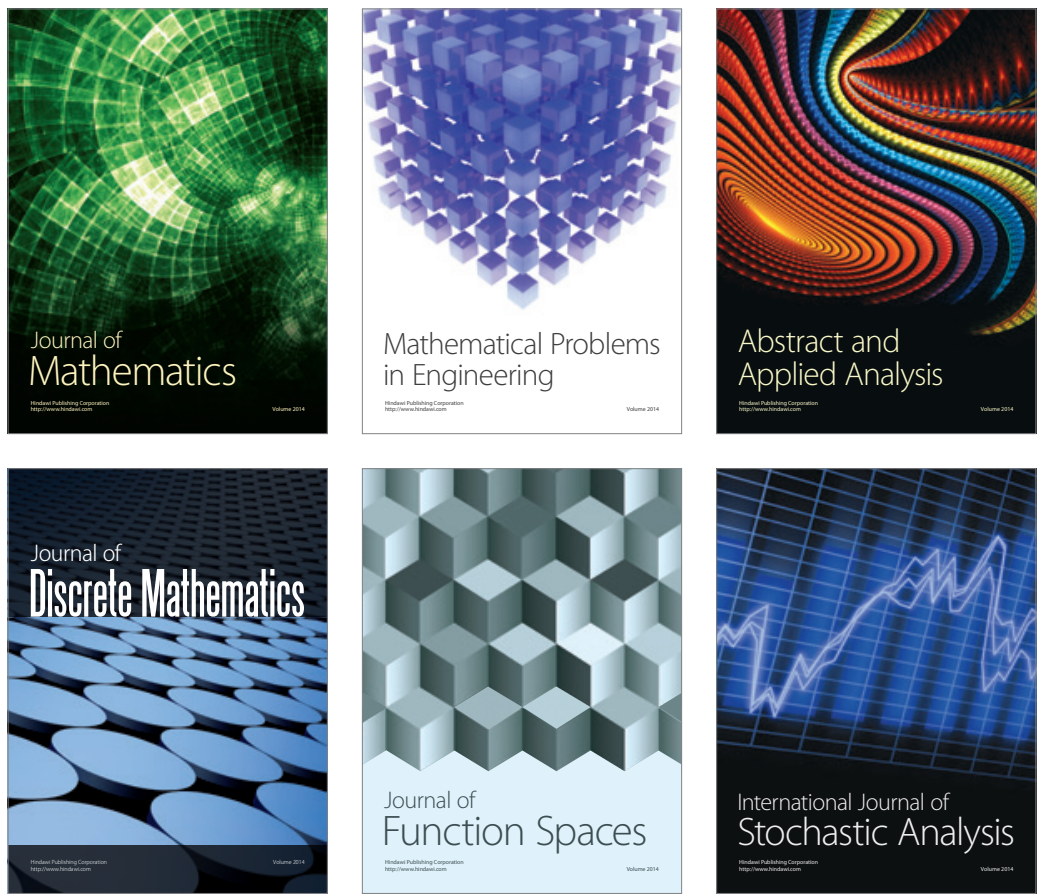

Journal of

Function Spaces

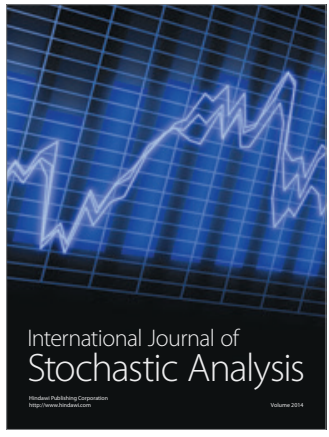

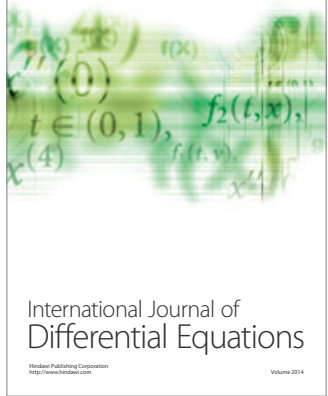
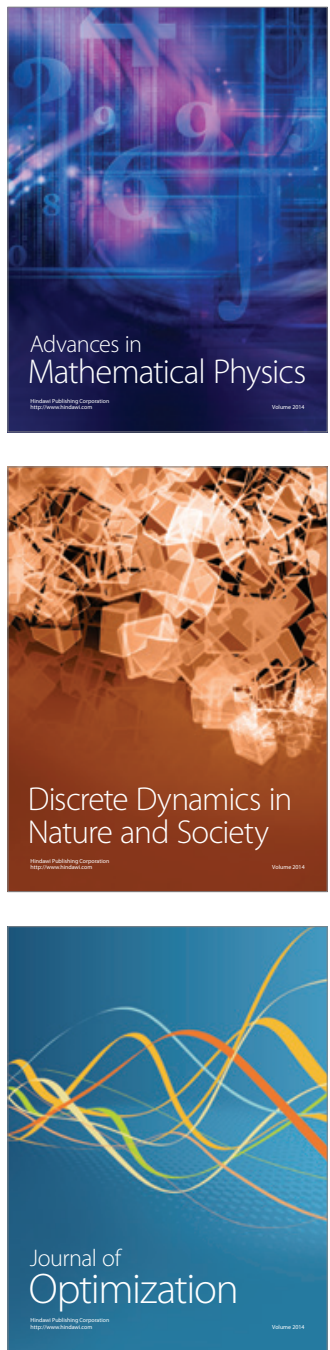